$$
\begin{aligned}
& \text { LES } \\
& 1944 \text { A } \\
& \text { U6S? }
\end{aligned}
$$

THE STARK EFHECT OF HELIUM IN SOME B TYPE STARS.

$$
\text { by }
$$

Anne B. Underhill

A thesis submitted in partial fulfilment

of the requirements for the degree of

Master of Arts in the Department of Physics.

The University of British Columbia

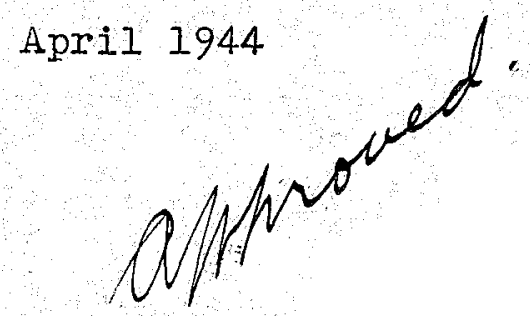




\section{TABLT OF CONTENTS}

Page

Classification of the Stars

According to spectral type $\quad 1$

According to luminosity type 2

Facts of Stark Effect

Outline of the Problem 6

Paper by Foster and Douglas 1934 6

Paper by Struve $\quad 1938 \quad 6$

Paper by Foster and Douglas $1939 \quad 7$

Paper by Goldberg $\quad 1939 \quad 9$

Observational Material

Stars studied $\quad 11$

Data obtained $\quad 12$

Results $\quad 16$

Confirmation of hypothesis by Foster and Douglas $\quad 16$

Identification of blend at $4144 \quad 18$

Identification of blend at $4026 \quad-19$

heconReonstpuctionxoecexeoted uhelinm line interisities in dwarf and intermediate stars

Summary of Results

Appendix

Tables of equivalent width measures for each line studied 


\section{TABLE OF CONTENTS-continued}

\section{$\underline{\text { Page }}$}

Appendix

Tables of equivalent width measures for cen $3 \mathrm{hn}$ each line studied

67 ophiuchi 31

$\gamma$ Pegasi 33

$\rightarrow$ Herculis $\quad 34$

$\eta$ Ursae Majoris $\quad 37$

Bibliography $\quad 38$ 


\section{THE STARK EFHECT OF HELIUM IN SOME B TYPE STARS}

Some years ago, a rather anomalous broadening of the HeI diffuse series triplet line $\lambda 4471$ became apparent, when attempts were made to establish the presence of Stark Effect in the spectra of dwarf B type stars by means of the broadened helium diffuse series lines., several hypotheses have been put forward to explain this feature, but because little quantitative work on the intensities of helium lines in B type spectra has been done, it has not been possible to check these theories. The purpose of this investigation is to provide data for this purpose by careful intensity measurements of the profiles of helium lines on high dispersion plates. Six typical B type stars with strong helium lines have been selected for study.

\section{Classification of the Stars:}

Stars are classified according to the main features of their spectra. The spectral types as defined by the Harvard criteria are named O, B, A, F, G, K, M, R, N, S, and depend chiefly upon the various degrees of ionization in the stellat atmosphere. B type stars, with temperatures around $20,000^{\circ} \mathrm{K}$, are amongst the hottest stars in the heavens. The chief absorption lines in their spectra are the HeI lines which reach a maximum intensity in this class, and the Balmer lines of hydrogen. There are a few relatively weak lines from the $10 n$ ionized atoms of several of the heavier elementy, but on the 
whole a B type stpectrum is relatively free of all lines except those due to netutral helium and hydrogen.

The stars of a given spectral type are further classified according to their absolute magnitude, or intrinsic luminasity. Those of high lubinosity are called giants, those of low luminosity dwarfs, while those of intermediate luminosity are called intermediates. As originally applied by Hertzsprung, these designations only referred to differences in absolute magnitude. However, subsequent work has showr that giant stars are, as the name implies, truly large stars with diameters up to $800 x$ the sun, although their masses do not increase proportionalely. Giant stars therefore have very low densities, and extremely tenuaus atmospherez. Dwarf stars are small, dense stars, -often smaller than the sun, with relatively dense atmospheres. Theirr surface gravity is on the average about 30 times the value of $g$ on the earth.

The spectra of dwarf and giant stars of the same spectral class are somewhat different, the lines of hydrogen and helium particularly tending to have extensive wings in the dwaris, but being sharp in the giants. Thess is in the absence of rotation of the star, for rotation broadens all spectral lines. The spectral type depends upon the average degtee of ionization in the stellar atmosphere. Low density and high temperature fagour ionization, hence dwarf stars of a given spectral type are at a higherrtemperature than giant stars of the same type. This higher temerature in dwarf atmospheres increases the Doppler broadening of the lines by the indiviaual moving 
atoms. Collisions between radiating atoms are also more frequent in the denser atmospheres of dwarf stars, hence collision is another factor tending to broaden the spectral lines. A third casue of broadened lines is Stark Effect. Owing to the relatively great density of electrons and jons in the atmosphere of a dwarf star, the absorbing and emitting atoms are in constantly changing electric fields. In these fields stark components of a line appear, but since the fields are constantly changing the net result is a superposition of all possible components, and hence a broadening of the spectral line. Struve and Elvey ${ }^{1}$ explained the greatly broadened wings of the hydrogen Balmer lines on this basis, and also some of the broadening of the HeI diffuse Iines. stark broadening is not noiceable in the lines from elements of higher atomic number since the displacements are too small. There is some evidence for the broadening of NI lines in stellar spectra, but on the whole Stark broadening is only appreciable in the bydrogen Balmer lines and the neutral helium diffuse series lines.

1. C. T. Elvery and 0. Sttruve, Ap. J. 12, 277, 1930. 
Facts of Stark Effect:

The main facts regarding stark Effect in helium lines are as follows. 2,3

There is no simple relationship between displacement of a Iine from its zero-field position and the strength of the field. The relationship is linear only after the displacement becomes large with repect to the separation of the sub-levels chacterized by different talues of the quantum number $\boldsymbol{l}$. Since the fine structure separations for helium are much larger than for hydrogen, the Stark displacements do not become linear until high fields are reached.

The sharp series and principal series of helium are displaced towards the red, the displacements increasing with serial number. The diffuse series lines are also shifted toward the red, and these displacements are much larger than for any of the other series.

Larger fields are not required for the production on electric combination lines, 200 volts/cm sufficing in some cases. The forbidden, or electric combination line, is usualIy displaced toward the violet although a red shift may occur at low fields and persist till moderate fields are reached. This may be seen in the behaviour of the $2 \mathrm{p}-5 \mathrm{f}$ line, $\lambda 4025$. Until the field reaches about $30 \mathrm{kv} / \mathrm{cm}$ the line movexs to the red, above that it reverses diretionarabmove sotbthelvilet.

2. J.S. Foster, Proc. Roy. Soc. 111, 137, 1927.

3. J. S. Foster and A.V. Douglas, M.N. 99, 150, 1939. 
The line 2p-4f, $\lambda 4470$, however, moves to the violet in all fields. The corresponding singlet lines beheve in the same way as the triplet lines.

The intensities of Stark components do not always increase with increasing field; in some cases the line disappears, reappearing at higher fields. In emission the total intensity of the forbidden components and the permitted line equal the intensity of the pernitted line at zero field.

In a stellar atmosphere a uniforr field is not expeeted. However the motion and density of charged particles will. create fluctuating fields which will have a permanent statistical effect. Fields of around $10^{3}-10^{4} \mathrm{v} / \mathrm{cm}$ have been considered reasonable by Struve ${ }^{4}$ and others. The electron partial pressure $p^{\prime}$ is considered to vary with $g$, the surface $g$ gravity following the formula $p^{h} g^{m}$. Struve considers $m=1 / 2$ a likely value ${ }^{5}$. In any case it is evident that so long as $m$ is positive, the greater $g$ is, the greater the electron pressure, and the electric fields produced. Hence one would expect Stark effect to be present in dwarf stars and negligible in giants. Also increased pressures produce broadened energy levels.

4. 0. Struve, Ap.J. 69, 173, 1929.

5. 0. Strure, Ap.J. 69, 173, 1929. 


\section{Outline of the Problem:}

In 1934 J. S. Foster and Miss A. V. Douglas 6 analysed profiles of He lines in the spectra of some $B$ type stars, and found very broad absorption lines near the normal positions of the aiffuse series (singlets and triplets) of helium. The profiles are somewhat complex, and the absorption is greater on the violet half of the line. In Stark effect the diffuse lines are displaced to the red only, except the first member which is displaced very slightly towards the violet. The whole displacement increases with serial number, but does not vary directly with the electric field. Assuming the presence of Stark effect Foster and Douglas were able to explain qualitatively most of the absorption in the red half of the Iine. The presence of electric combination lines $(2 p-4 f$, $2 p-5 f$, etc) explained part of the violet wing, but these lines did not have the right wavelengths or intensities to explain the total absorption, and a residual absorption was

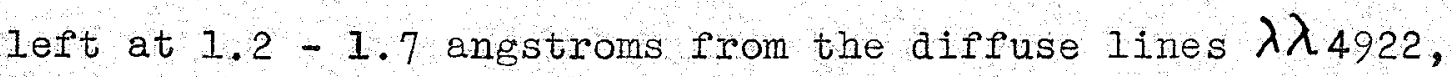
$4471,4388,4026$.

In a paper 7 published in 1938 struve studied the residual absorption near the Iine $\lambda_{4471}$ in the intermediate star $\alpha$ Herculis. He identiried the violet wing as being chiefly $\lambda 4470$, the electric combination line $2 p-4 \mathrm{f}$. The ordinary Stark Effect shifts the 2p-4d Iine ( 14471 ) towards the red;

6. J.S. Foster and A.V. Douglas, Nature 134, 417, 1934.

7. 0. Struve, observatory 61, 53, 1938 . 
the $2 p-4 f$ line (zero-field position $\lambda 4470$ ) is shifted towards the violet. Hence the lines should spread apart. If present, the separation of these lines could be detected on the high dispersion plates used by Struve. The profiles do not indicate any such effect. To explain this filling in between the lines, Struve considered the effect of collisional damping. By following a traif of reasoning developed by Russel and Unsold, Struve found that the results of this one Ine $(\lambda 4471)$ could be explained, if in dwarf stars there is a damping constant for the He diffuse series which is approximate1) twenty times larger than the darning constant for other lines. He found that this damping constant depends upon the sensitivity of the given line to stark Effect. He found also that this type of broadening is independent of the actual pattern of the Stark Effect, and will produce wings in relativeIy weak lines fully accounting for their symmetry. The symmetrical collision contour is superimposed over the unsymmetrical contour produced by the blended Stark effect, giving the net profile. Only the Iine $\lambda \$ 471$ is considered. In giants there should be no marked difference in the damping constant from its ordinary value owing to absence of electric fields.

In 1939 Foster and Miss Douglas ${ }^{8}$ studied helium line profiles in the stars $\mu$ Herculis and $\gamma$ Pegasi, and tried to account for the observed broadening of the HeI diffuse series lines both quantitatively and qualitatively by Stark Effect.

8. J.S. Foster and A. V. Douglas, M.N. 92, 150, 1939. 
Near $\lambda 4922$ and $\lambda 4471$, they found areas of absorption which they could not explain by pure Stark effect, or by likely blends. These areas in each case totalled very nearly $50 \%$ of the line. To explain this absorption, Foster considered a possible cycle as indicated in fig. 1 , and reasoned as follows:

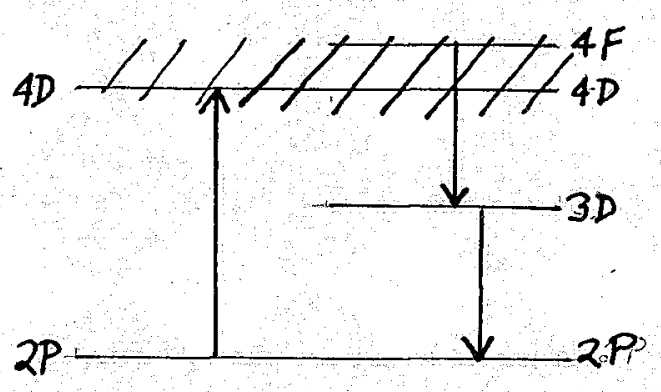
If stellar conditions are such as to give diffuse lines 3 angstroms or more wide, and there is a reasonable departure from the Boltzman distribution, the broadening of the $4 D$ level might be expected. When radiation characteristic of the zero-field position of the electric combination line $2 \mathrm{p}-4 \mathrm{f}$ is considered, events in the direction indicated are more probable than in the reverse direction 9 . Thus even if there were no electric fields present to produce the $f$ line (this could not be so, since conditions giving rise to the broadened, levels would also give rise to electric fields), there would be relatively streng $2 p-4 d$ absorption in the region of the fline, since emission accompanying the absorption will be relatively weak here due to a leakage $4 \mathrm{~d} \rightarrow 4 \mathrm{\rho} \rightarrow 3 \overline{\mathrm{a}} \rightarrow 2 \mathrm{p}$. In varying fields it would be expected that the $2 p-4 f$ line be augmented at zero-fild positions with its maximum intensity shifted toward the d line, and there should be no sharp distinction between the lines.

9. S. Rosseland, Ap.J. 63, 218, 1926. 
This absorption is thus a secondary maximum in the d absorption and its existence is not proof that eletric fields exist in stellar stmospheres. That such fields do exist however is evident from the greatiy extended Balmer wings, and the broadened wings of the sharp series and diffuse series HeI lines.

As a direct consequence of a leakage of this sort, the lines $3 d-4 f$ and $2 p-3 d$ should be weakened by emission more than is the case in a normal star, i.e. any star in which conditions are not likely to make such a eycle possible. These conditions are most likely to be met in a dwarf or intermediate star, but not in a giant.

The 3d-4f line cannot be easily observed since it is in the infra red, 18693.4 antstroms for the singlets and 18684.2 angstroms for the triplets. However the $2 \mathrm{p}-3 \mathrm{~d}$ line can be observed as it is 6678 angstroms for the singlets and 5876 for the triplets.

In a paper ${ }^{10}$ published in 1939 Goldberg also considers the problem of the abnormally large absorption near $\lambda 4470$. He shows by a consideration of transition probabilities, and the curve of growth effect, that fields necessary to make the electric combination line $\lambda 4470$ strong enough to take in all the observed absorption in cHerculis are not exceptionally large, but just what might be expected in the atmosphere of a dwarf star. He does not consider what displacement of $\lambda 4470$ would occur 10. L. Goldberg, Ap.J. $89623,1939$. 
because of these fields, but is content with saying that the observed absorption can be quantitatively explained by simple Stark absorption. He does not consider what might be the effect on any other of the diffuse lines.

The object of the present investigation is to examine spectral data, and obtain evidence in support of, or against, the Foster hypothesis. A decided weakening in absorption of the first member of the singlets and triplets will support Foster's hypothesis, and if there is no observable weakening of the fijst member, then it does not seem likely that Foster's hypothesis is correct. 
Observational Material:

\begin{tabular}{|c|c|c|c|c|}
\hline Star & HD No. & $m_{v}$ & $\begin{array}{c}\text { Spectral } \\
\text { Type }\end{array}$ & $\underset{\text { Type }}{\text { Luminosity }}$ \\
\hline$\gamma$ Pegasi & 866 & 2.9 & $\mathrm{~B} 2.5 \mathrm{ss}$ & intermediate \\
\hline P Leonis & $91316^{\circ}$ & 2.9 & B1. Os & giant \\
\hline$\eta$ Ursae Majoris & 120315 & 1.9 & B5. Onn & dwarf \\
\hline$\iota$ Herculis & 160762 & 3.8 & B3. 5 & intermediate \\
\hline 67 Ophiuchi & 164353 & 3.9 & $\mathrm{~B} 5.0 \mathrm{~s}$ & giant \\
\hline 55 Cygni & 198478 & 4.9 & $B 3.0 e \alpha$ & giant \\
\hline
\end{tabular}

Intensity measurements were made to verify the spectral classification. The results obtained by Dr. R. M. Petrie were in accordance with the spectral types assigned by Williams 11 .

11. E.G. Williams, Ap.J. 83, 305, 1936. 
Table 2.

Instruments Used

\begin{tabular}{|c|c|c|c|}
\hline Instrument & $\begin{array}{c}\text { Dispersion at } \\
5876 \text { in A/mm }\end{array}$ & $\begin{array}{c}\text { Dispersion at } \\
\text { H } \gamma \text { in A/mm }\end{array}$ & Range covered \\
\hline I M & & 30.1 & $4922-3820$ \\
II I & & 11.0 & $4471-4009$ \\
III M & 30.3 & 10.5 & 5876 \\
III R & 30.3 & 10.5 & $6678-4922$ \\
III I & & 7.2 & $4922-4009$ \\
Litt II & 29.4 & 8.7 & $6678-4009$ \\
IItt GI & 14.6 & 14.7 & $6678-3820$ \\
IItt GIII & & 4.48 & $4388-3820$ \\
GI M & 21.9 & & $6678-5876$ \\
GI I & 15.6 & & $6678-5876$ \\
GII L & 5.1 & & 5876 \\
\hline
\end{tabular}

The plates were taken at the Dominion Astrophysical Observatory by various members of the staff. Most of the plates were calibrated for intensity work by the rotating sector now in use, although a few of the plates taken some jears ago were calibrated by the neutral tint wedge which was in use at that time.

Microphotometer tracings were made of the HeI diffuse series Iines $\lambda \lambda 6678,5876,4922,4491,4388,4144,4026,4009$, 3820. Line profiles were obtained using the intensitometer devised by Dr. C. S. Beals, and the equivalent widths of the lines derived. Plates I and II show typical profiles as obtained by the intensitometer. The two profiles taken from plates of Ypegasi clearly show much complex structure which 


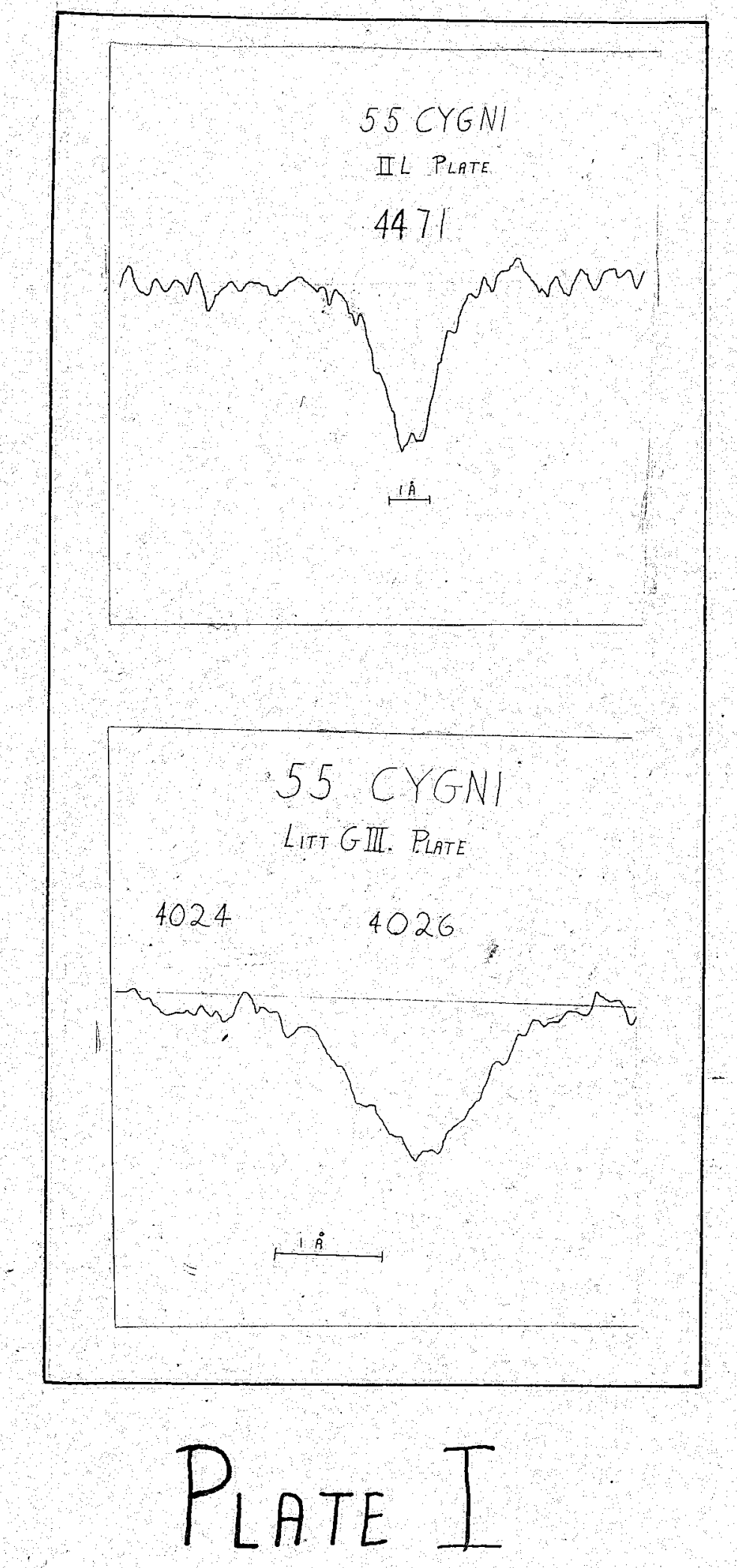




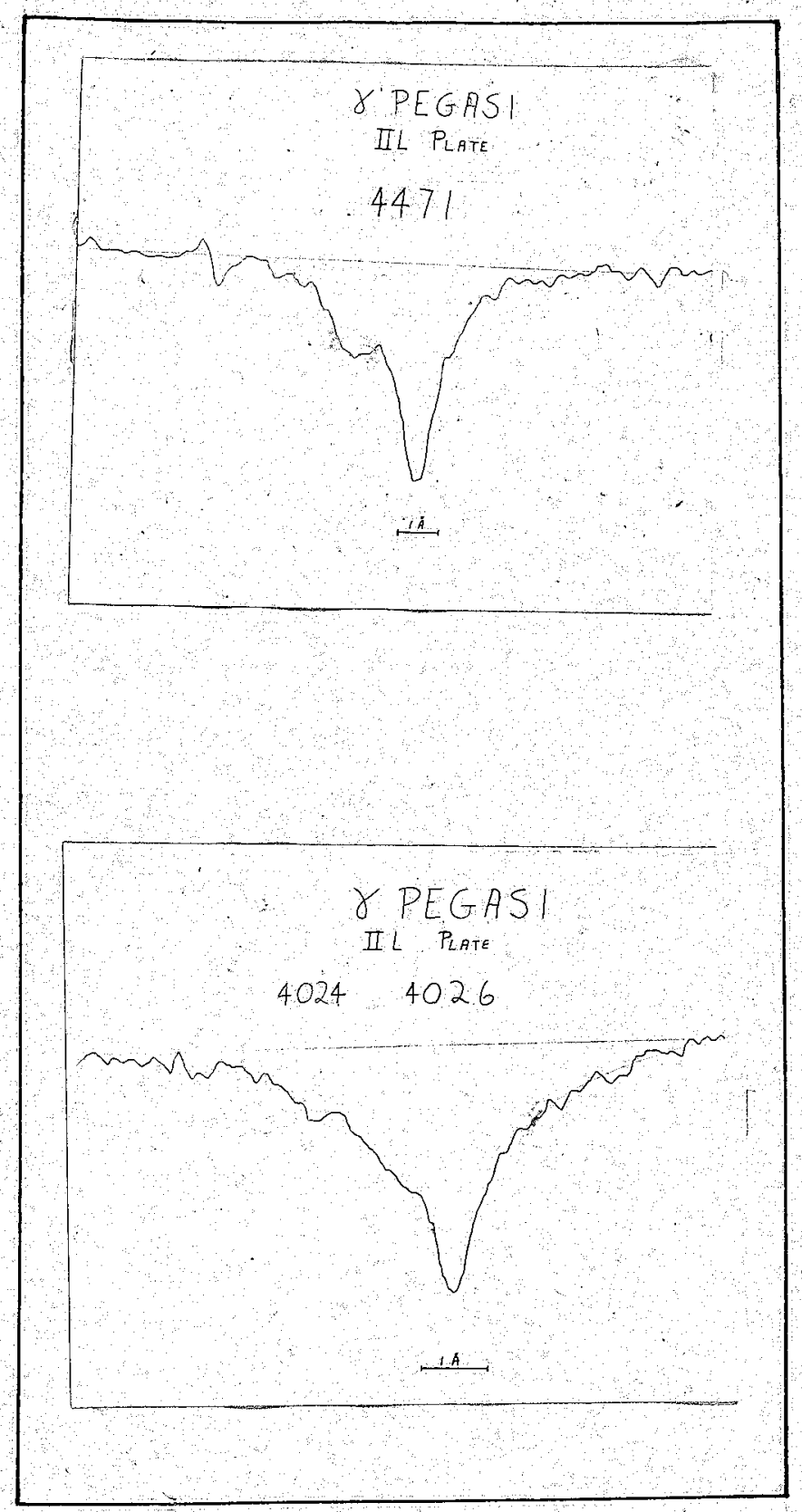

Plate II 
is not present in the profiles of lines in 55 cygni, a giant star. Note the resolution of the two helium lines $\lambda 4026$ $\left(2^{3} \mathrm{P}-5^{3} \mathrm{D}\right)$ and $\lambda 4024\left(2^{I_{P}}-7^{I} \mathrm{~S}\right)$ on the Litt GIII Plate of 55 Cygni. The dispersion here is $4.64 \mathrm{~A} / \mathrm{mm}$. In intermediate and dwarf stars the region between these two lines is affected by absorption by the electric combination line $\lambda_{4025}$.

The mean equivalent width for each line is shown in the following tables. The separate determinations of equivalent width for each line together with the instrunent used are shown in tables in the appendix.

The equivalent wiath of a line is a measure of its intensity. An absorption line ine general does not completely absoub the continuous background, but only weakens it. The lines are in general $\mathrm{V}$ shaped, having more or less extensive wings, depending upon the conditions giving rise to the line. The equivalent width of a line is the energy absorbed by the line. This may be calculated if the line is considered as a rectangle with height equal to the continuous spectrum and area equal to that of the natural profile. The energy enclosed $\mathrm{w}=\int_{\lambda_{1}}^{\lambda_{2}} E_{\lambda} d \lambda$ where $E_{\lambda}$ is the Planck Radiation Law function $8 \pi c h \lambda^{-5}\left(e^{\lambda_{1}} \frac{h}{\lambda k T}-1\right)^{-1}$. over short ranges $E_{\lambda}$ is constant hence $W=E_{\lambda}\left(\lambda_{2}-\lambda_{1}\right)$. In practice when intensities are to be compared, but the actual energies absorbed are not required $E_{\lambda}$, the height of the contifuous spectrum, is called unity. Hence the intensities of the lines are expressed in equivalent angstroms, i.e. $\left(\lambda_{2}-\lambda_{1}\right)$. 
Table 3. Mean Equivalent Widths (Giants)

\begin{tabular}{|c|c|c|c|}
\hline Iine & $\rho$ Leonis & 55 cygni & 67 ophiuchi \\
\hline 6678 & 1.02 & 1.00 & 1.16 \\
4922 & .47 & .64 & .74 \\
4388 & .36 & .49 & .41 \\
4144 & .30 & .43 & .45 \\
4009 & .21 & .30 & .34 \\
5876 & 1.19 & .83 & .97 \\
4471 & .71 & .74 & .73 \\
4026 & .51 & $.62^{13}$ & .64 \\
3820 & .33 & .56 & .65 \\
\hline
\end{tabular}

12.5876 is possibly weakened by emission in 55 cygni and 67 ophiuchi. 55 cygni is classified as an emission line star.

13. The helium sharp series Iine $\lambda 4024$ has been subthacted from th the measured profiles of $\lambda 4026$ in the giant stars 55 Cygni and 67 ophiuchi. 
Table 4. Mean Equivalent Widths (Dwarfs)

\begin{tabular}{|c|c|c|c|}
\hline D Line & $\gamma$ Pegasi & r Herculis & 证sae Ma joris \\
\hline 6678 & .55 & .47 & .54 \\
4922 & .96 & .91 & .85 \\
4388 & .82 & .64 & .70 \\
4144 & .79 & .62 & .66 \\
4009 & .55 & .44 & .43 \\
5876 & .77 & .61 & .80 \\
4471 & 1.28 & 1.07 & 1.01 \\
4026 & 1.25 & 1.02 & 1.28 \\
3820 & 1.14 & .93 & $.60^{14}$ \\
\hline
\end{tabular}

14. In $\eta$ Ursae Majoris the wings of Hn and Ho overlap owing to the great broadening of the profiles by the rapid rotation of the star and development of Stark wings. The height of the contiuous spectrum probably was drawn too low for $\lambda 3820$ because of their overlapping. 

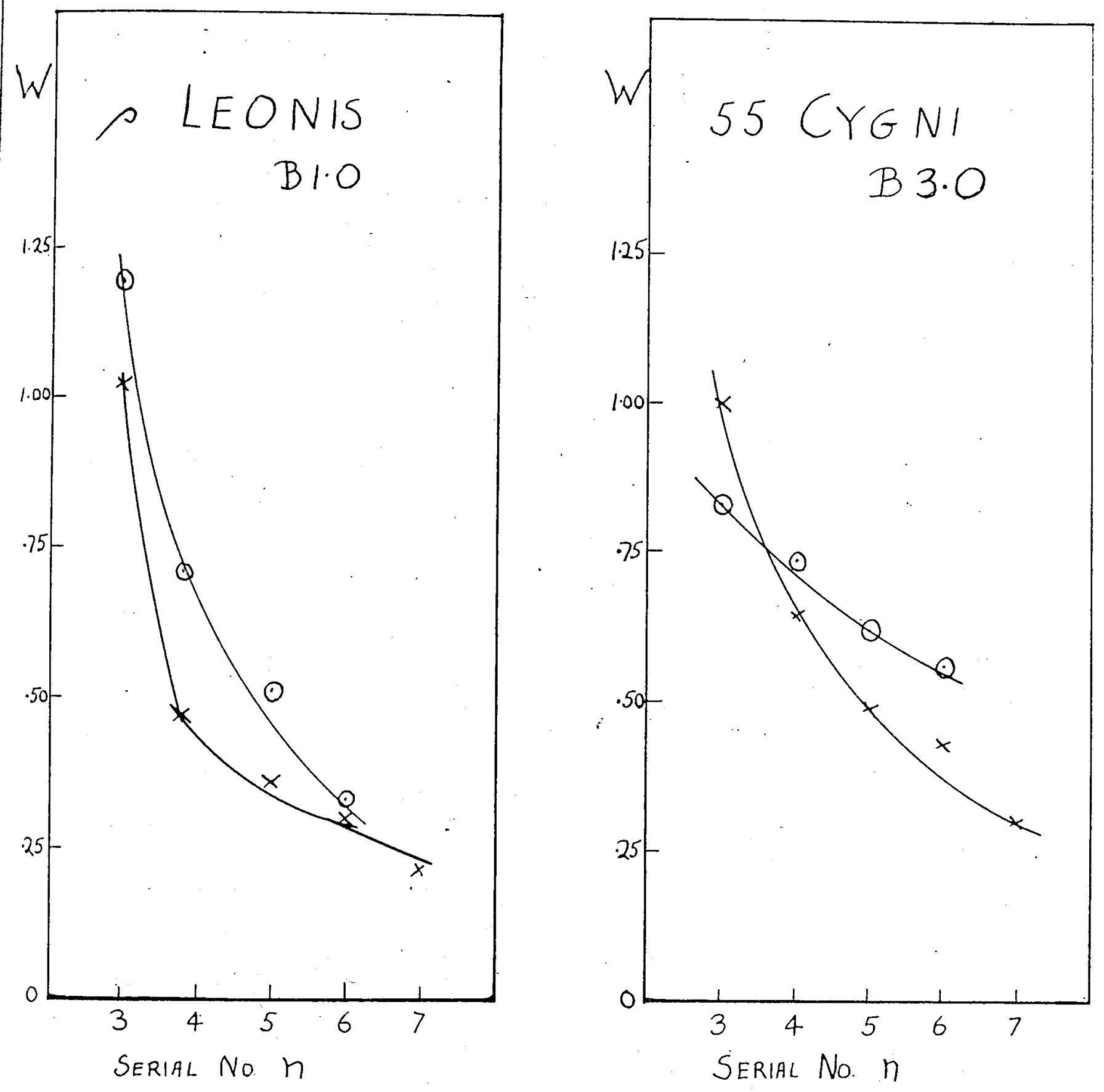

fig 2

$$
\begin{aligned}
& x=\text { SINGLETS } \\
& \odot=\text { TRIPLETS }
\end{aligned}
$$

fig 3 


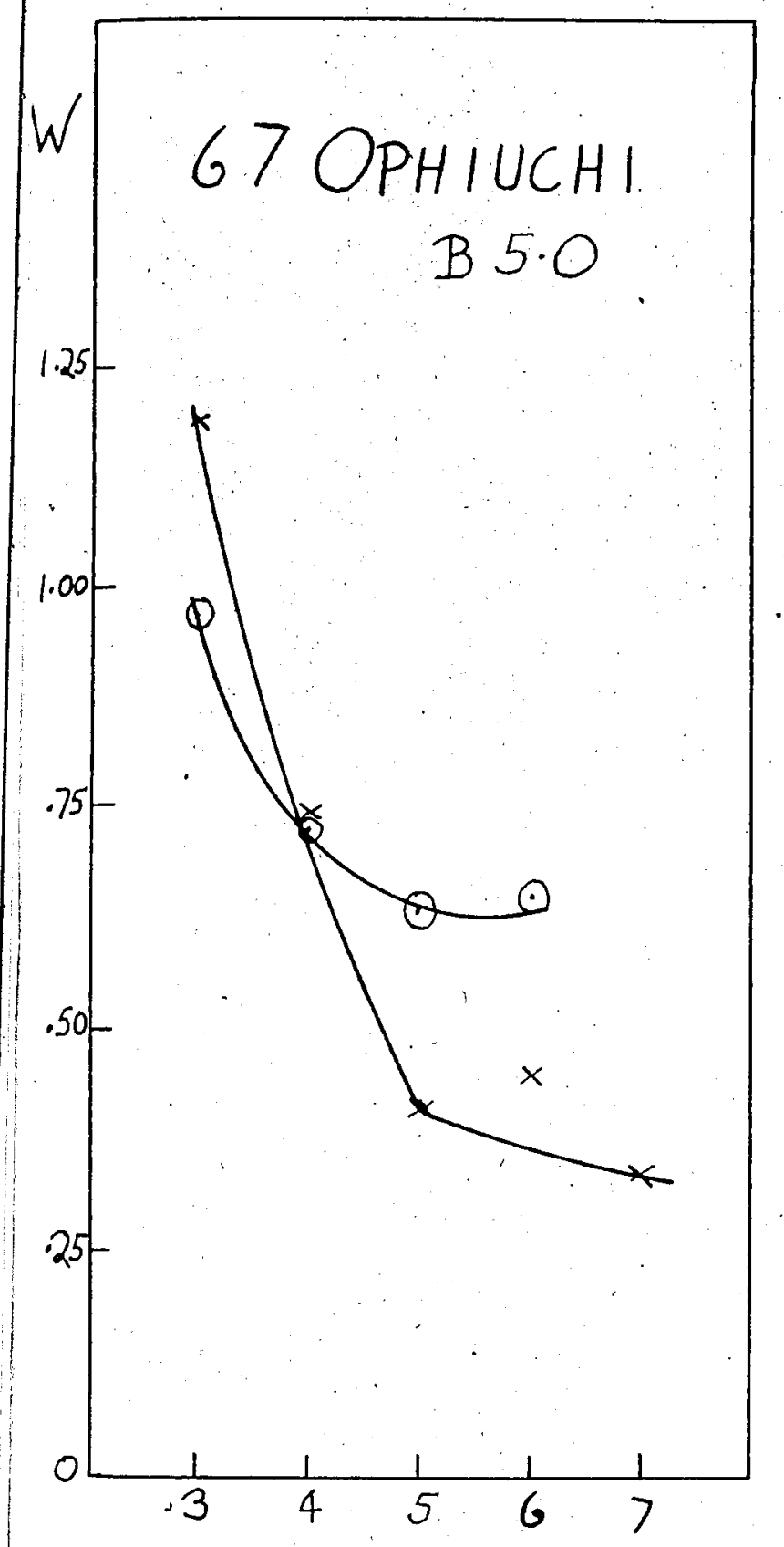

SERIAL No $n$

fig. 4.

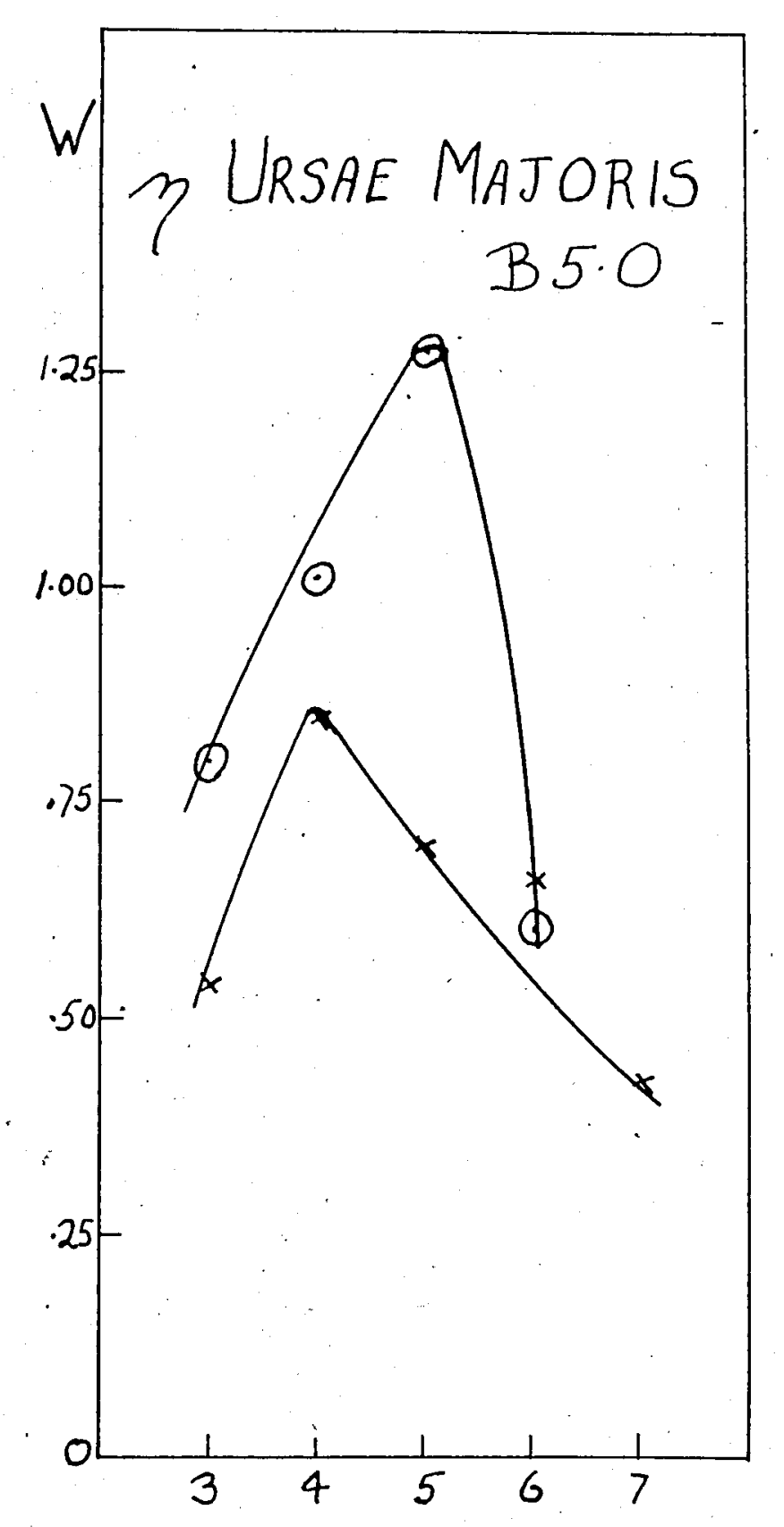

SERIAL No. $n$

fig. 5

$$
\begin{aligned}
& x=\text { SINGLETS } \\
& \odot=\text { TRIPLETS }
\end{aligned}
$$




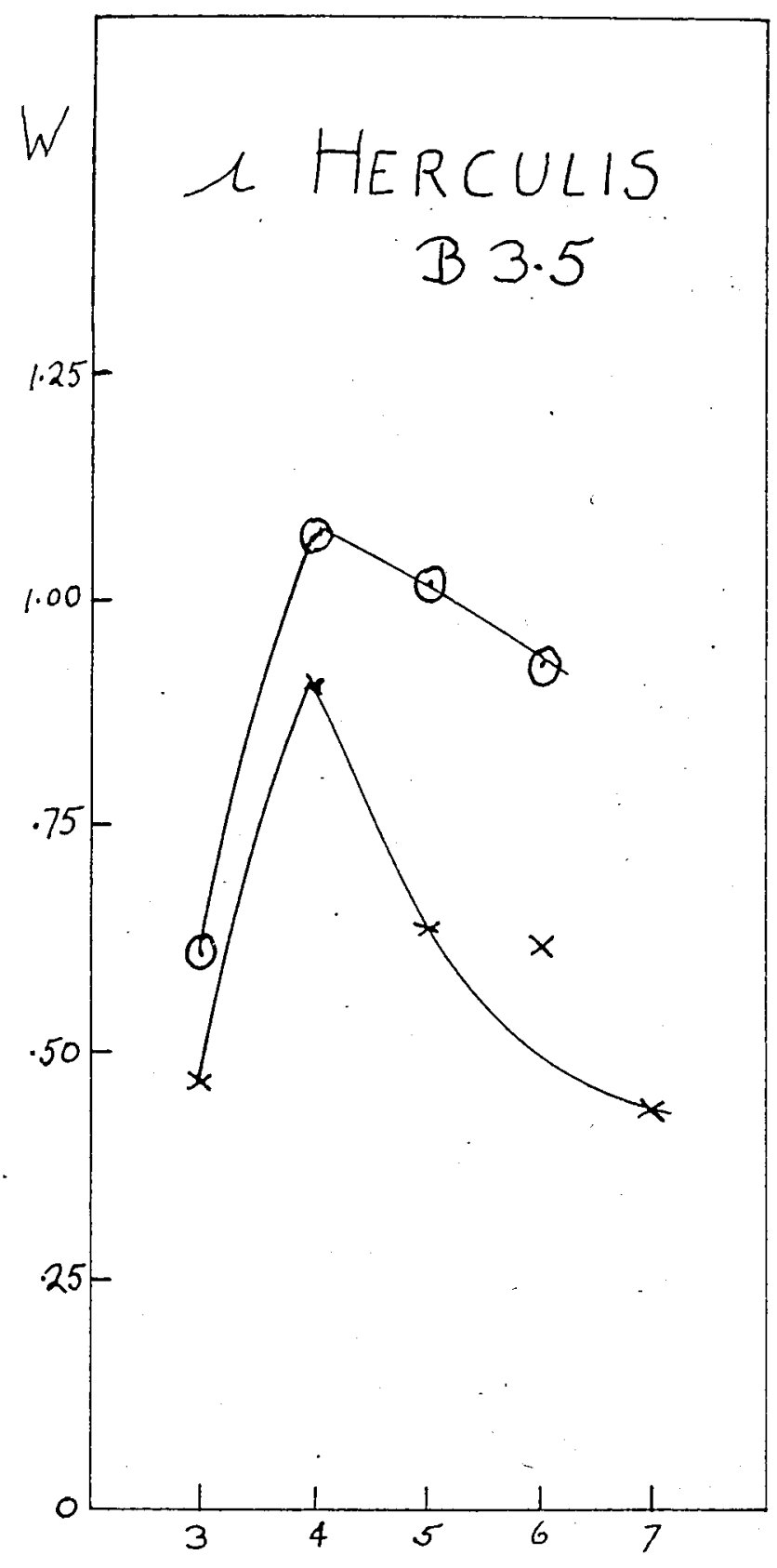

Serial No. $n$

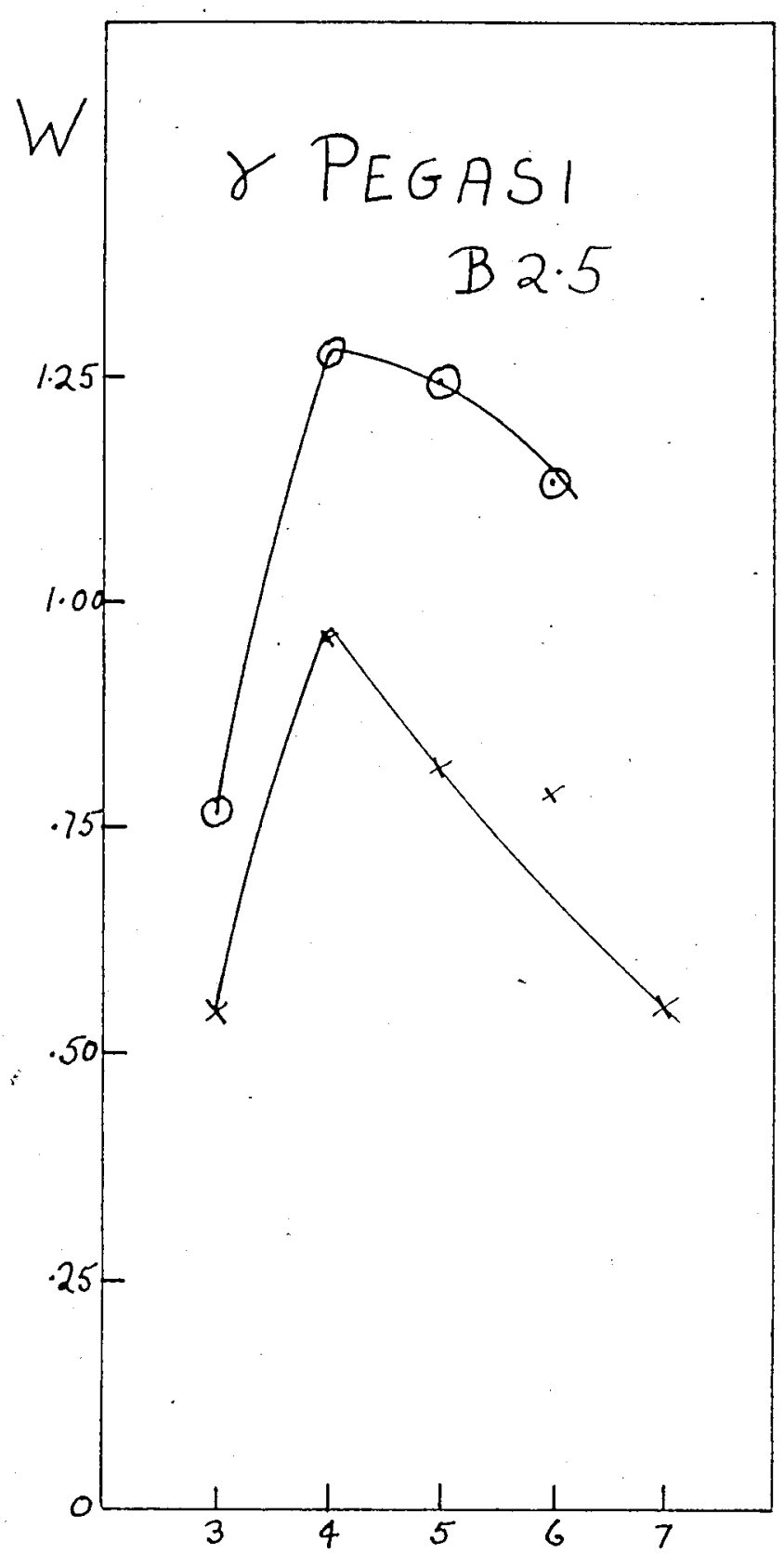

Serial No. h

fig. 6.

$$
\begin{aligned}
& X=S I N \mid G L E T S \\
& \odot=T R I P L E T S
\end{aligned}
$$

fig. 7 . 


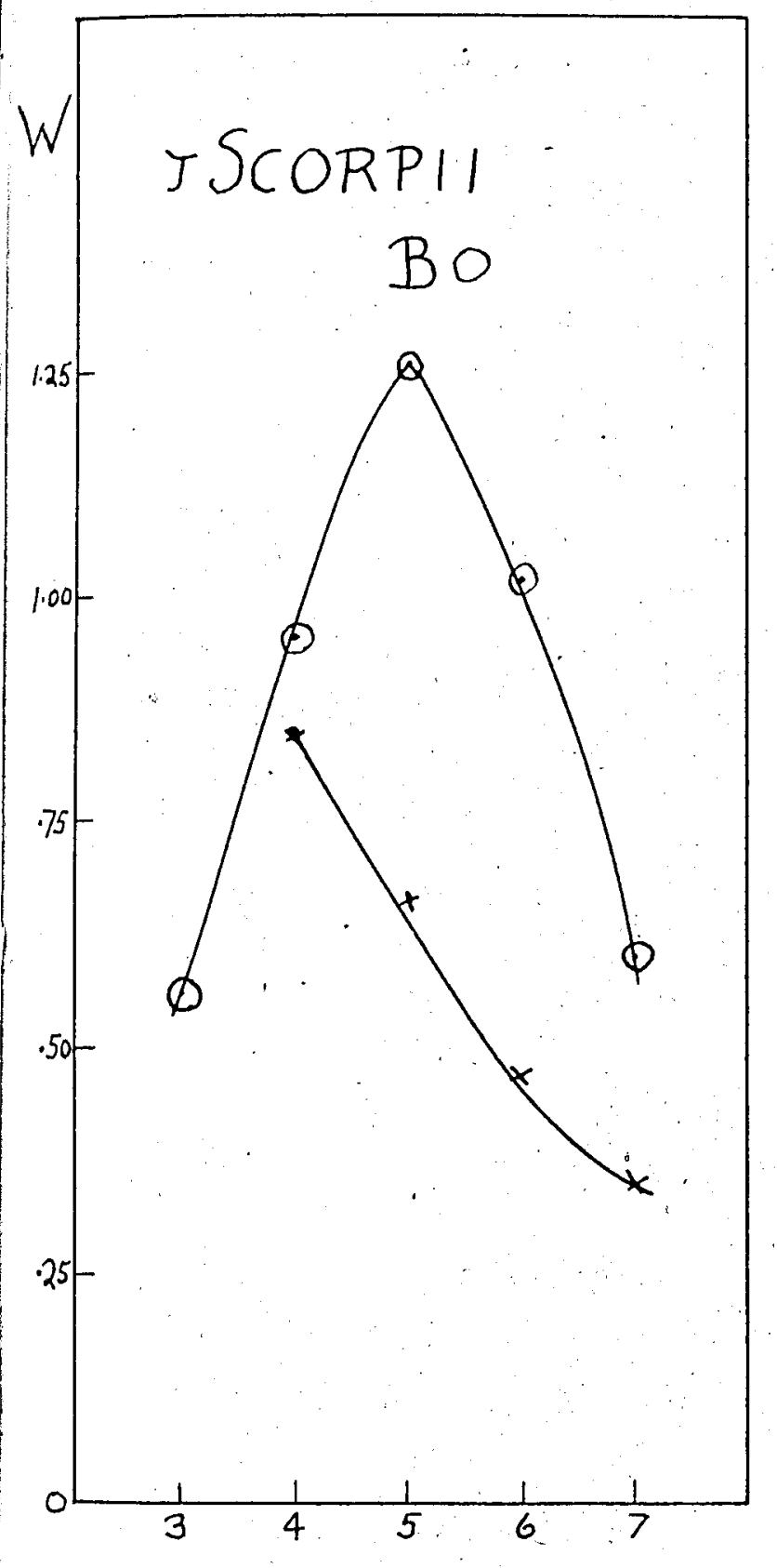

SERIAL No. h

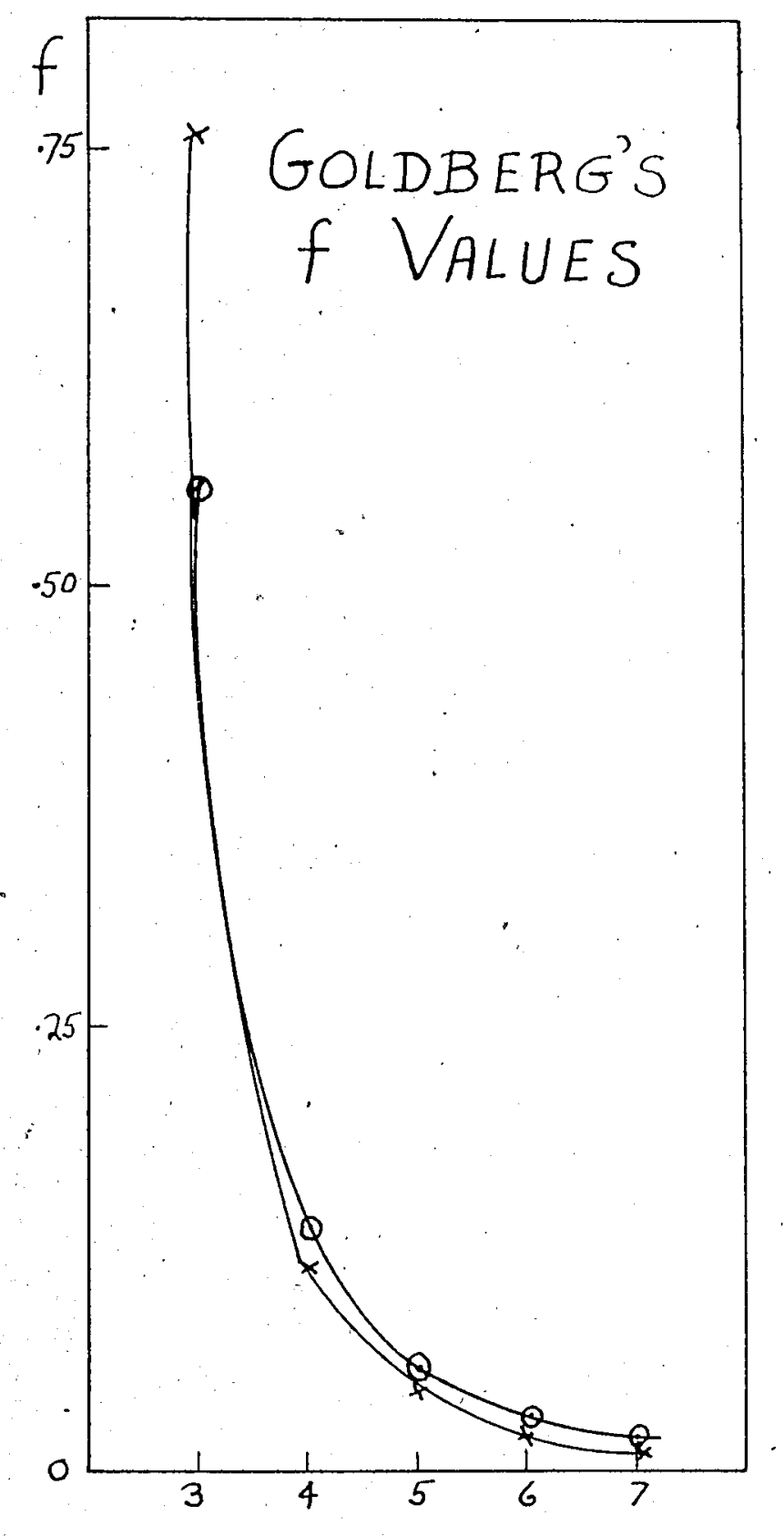

SERIAL No. $n$

fig. \&

$$
\begin{aligned}
& X=\text { SINGLETS } \\
& \boldsymbol{\Theta}=\text { TRIPLETS }
\end{aligned}
$$$$
\text { fig. } g \text {. }
$$ 


\section{Results:}

From a consideration of figures $2-7$ which are a plot of serial number against equivalent width for lines of the diffuse series of helium, a difference between giant stars, intermediates, and dwarfs is at once apparent. In the giants, pleonis, 67 ophiuchi, and 55 Cygni, the fizst member of both the singlet and triplet series is the strongest line of the series, and the intensities of the lines decrease with serial number much as the $f$ values calculated by Goldberg ${ }^{15}$ (see fig. 9). The first members are not relatively so strong as the first $f$ values, but some incipient emission in these lines might be expected as the stars are of early B type.

In the intermediate and dwarf stars, $\gamma$ Pegasi, Herculis, and Ursae Majoris, the first member of each series is one of the weakest lines measured, the second member being much stronger. Stark effoct broadens the second and succeeding members but does not affect the first member. The first member should therefore have relatively the same strength as in a giant star of the same spectral class or one of slightly earlier class. That this is not the case is evident.

When the various hypotheses put forward to explain the relatively large absorption near the second nember of the singlets $(\lambda 4922)$ and of the triplets $(\lambda 4471)$ are considered, it is seen that a weakening of $\lambda 6678$ and $\lambda 5876$ is a direct consequence of the cycle proposed by Foster and Douglas. The intensity of a spectral line is a function of the 15. Golaberg, Leo, Ap.J. 90, 414, 1939. 
number of atoms absorbing a given wavelength and emitting it. If more atoms are absorbing than emitting, then the line is an absorption line. If the equilibrium is shifted so that more atoms are emitting energy of the given wavelength than absorbing it, the line will be an emission line. The equilibrium between absorption and emission, and hence the strength of the line, is governed by the temperature and density of the star's atmosphere. If some other effect causes an emission or absorption of energy apart from the general equilibrium, then the line will be strengthened or weakened accordingly. This is what occurs in the leakage cycle proposed by Foster and Douglas. Energy is absorbed at $\lambda 4470$ owing to level broadening, but it is not re-emitted there in proportion to the equilibrium rate in force, but is emitted at a higher wavelength, $\lambda 5876$. This emission at $\lambda 5876$ is not balanced by any absorption at this wavelenth, hence this line i.e. $\lambda 5876$ is weakened in absorption. Hecording to the explanations of the extra absorption at $\lambda 4470$ by struve and Geldberg, the leading members of the series should be unaffected, and hence have the same relative strength as in a ginant of the same or neighbouring classes. The observed weakening of $\lambda 6678$ and $\lambda 5876$ could not possiblyy be due to emission as ordinarily considered in emission line stars, for then it should be more noticeable in the giant than the intermediate or dwarf stars. It is therefore concluded that the observed weakening of the leading members, relative to the other lines of the diffuse series, is strong eviaence in favour of the cyclic transitions suggested by Foster and Douglas. 
A similar plot of data secured by Unsdid ${ }^{16}$ on TScorpil, a dwarf BO star, shows the same weakening (fig. 8). It is also interesting to note that H.H. Plaskett ${ }^{17}$ qualitatively noticed this weakening of the leading member of the series, when he studied the spectrum of 10 Lacertae, an 09 star classified by Williams as an intermediate. This weakening of $\lambda 6678$ and $\lambda 5876$ is also reported by E.G. Williams ${ }^{18}$ for the dwarfstars 7 Ursae Majoris and $\alpha$ Leonis.

A consideration of figs, 2-7 shows that in most cases the equivalent widths for $\lambda 4144$ and $\lambda 4026$ are above the smooth curve. The deviation in A.144 is larger in the later spectral types e.g. B5 ( 67 Ophiuchi), than the earlier ones e.g. BI $p$ Leonis). It seems likely that the extra absorption is due to a blend with sulphur lines as the profile of $\lambda 4144$ in 67 ophiuchi has broad sheil shallow wings, although this star is a sharp lined giant. of the elements mentioned by struve ${ }^{19}$ as likely to be represented in a B type speetrum, only oxygen and sulphur have lines in this region. Moreover the oxygen multiplet has very low intensity, whereas the sulphur multiplet lines have the following wavelengths and intensities. 20.

16. A. Uns81d, Zeit. f. Astrophys, 21, 22, 194I

17. H.H. Plaskett, Pub. D.A.0., 1, 325, 1922

18. E.G. Williams, Ap. J. 83, 279, 1936

19. 0. Struve, Ap. J. 24, 225, 1931

20. C.E. Moore, A Multiplet Table of Astrophysical Interest. 
Table 5 Sulphur Multiplet

\begin{tabular}{|cc|cc|}
\hline 4142.42 & 8 & 4168.37 & 5 \\
4145.05 & 8 & 4189.68 & 4 \\
4153.05 & 10 & 4277.16 & 4 \\
4162.64 & 10 & & \\
\hline
\end{tabular}

In table 6 are given the measured intensities of the only two lines of the supphur multiplet which are free from blends and which are clearly visible on moderate dispersion plates of the stars studied. The assumption that the relative intensities of the lines are the same in stellar spectra as those given by c. Moore, ${ }^{21}$ gives an estimate of the combined strength of the chiel lines causing the blend. When this amount is subtracted from the total equivalent width for $\lambda 4144$, the point lies much closer to the curve.

\begin{tabular}{|c|c|c|c|c|}
\hline & $\gamma \mathrm{Peg}$. & $55 \mathrm{cyg}$. & cHerc. & 670phi. \\
\hline Iine & $B 2.5$ & $B 3.0$ & $B 3.5$ & B5. 0 \\
\hline 4162 & .03 & $f$ & .01 & .05 \\
\hline 4153 & .07 & .05 & .04 & .06 \\
\hline According t & multiplet & table $4142+4$ & $145=.8(4153$ & -4162/giving \\
\hline $4142+4145$ & .08 & .04 & .04 & .09 \\
\hline
\end{tabular}

The most likely line blending with $\lambda 4026$ is the $2^{1} \mathrm{P}-7^{I_{S}}$ helium I line $\lambda 4024$. The preceeding members in this series can be seen on all the plates taken. On high dispersion plates of the giant star 67 ophiuchi $\lambda 4024$ is seen to be distinctly 21. C.E. Moore, A Multiplet Table of Astrophysical Interest. 
separated from 44026. In intermediate or dwarf stars, however, the Stark components cause a blend over this region. The meas ured equivalent widths of the two preceeding lines in the sharp series of Hel are given in table 7 , and the equivalent width of $\lambda_{4024}$ is estimated as above from the intensities given by c. Moore. When this amount is subtracted from the total measured equivalent wiath of $\lambda 4026$, the line is closer to the smooth curve. There is no imrediately obvious line of any other elements likely to be present which could cause such a blend.

Table 7 Measured Equivalent Widths

\begin{tabular}{|c|c|c|c|c|}
\hline & rPeg. & 55Cyg. & AHerc. & $670 \mathrm{phi}$. \\
\hline Iine & B2.5 & B3.0 & B3.5 & B5.0 \\
\hline 4437 & .14 & .11 & .14 & .08 \\
4168 & .10 & .06 & .07 & .05 \\
4024 & & .04 & & .13 \\
According to multiplet table 4024 & $.5(4168+4437)$ giving \\
4024 & .12 & .08 & .10 & .07 \\
\hline Average & .12 & .06 & .10 & .10 \\
\hline
\end{tabular}

However after this correction $\lambda 4026$ is still the most intense hellium line in the spectra of the two dwarf stars YUrsae Majoris and J Scorpii. Apparently some effect peculiar to dwarf stars is strengthening this line. When curves of growth (curves showing the relative increase of line strenth with increasing number of absorbing atoms) are plotted from the data for each star, the scatter around the curve for individual observations is small except for the lines $\lambda \lambda 4026,5876,6678$. The levels associated with the singlet line $\lambda 4388$, and the gorresponding 
tripdet line $\lambda 4026$ are very nearly of the same energy, so that one would expect any extra absorption in $\lambda 4026$ due to level broadening would also be found in $\lambda 4388$. Moreover, the curves of growth show that if anything $\lambda 4388$ is on a steeper part of the curve than $\lambda 4026$. Thus any increase in the number of absorbing atoms should have greater effect on the strength of $\lambda 4388$ than $\lambda 4026$. Actually it is $\lambda 4026$ which is alone strengthened to a degree nore than seems likely for pure stark broadening. Hence we may conclude that this is some effect peculiar to the $\lambda 4026$ Iine, and the observed discrepancies are not a curve of growth effect. It is unlikely a foster leakage would occur only in the tiplets, when the triplet and singlet levels are so close together.

Further data on dwarf stars covering the whole range of B stars from BO to B8 must be obtained before any definite conclusion ean be reached on this apparently excessitie strengthening of $\lambda 4026$.

Foster and Douglas in their analysis of the HeI diffuse series lines in $\mu$ Herculis and YPegasi, claim that the leakage at $\lambda 4922$ and $\lambda 4471$ amounts to well over $40 \%$ of the line. Since some of this energy is emitted again at $\lambda 6678$ and $\lambda 5876$ we can estimate the change produced in the equivalent width of these lines. If the continuous background of the spectrum is. considered to behave as black body radiation, it is possible to calculate what the change in equivalent width is at $\lambda 6678$ for a given change at $\lambda 4922$.

If there are $N$ transitions causing the leakage absorption 
at wavelenth $\lambda_{1}$ then the energy absorbed is,

$$
N h v_{1}=N h c \lambda_{1}^{-1}=W_{1} 8 \pi c h \lambda_{1}^{-s}\left(e^{\frac{h c}{\lambda, k T}}-1\right)^{-1}
$$

giving $\quad N=w_{1} 8 \pi \lambda_{1}^{-4}\left(e^{\frac{b}{n_{1}}}-1\right)^{-1}$

where $b=\frac{h c}{T}$ since for a given star $T$ is constant.

There are also N transitions emitting energy at $\lambda_{2}$, hence

$$
N h \nu_{2}=N h c \lambda_{2}^{-1}=w_{2} 8 \pi c h \lambda_{2}^{-5}\left(e^{\frac{h_{c}}{\lambda_{2}} k T}-1\right)^{-1}
$$

giving $\quad N=W_{2} 8 \pi \lambda_{2}^{-4}\left(e^{\frac{h c}{\lambda_{2}} k T}-1\right)^{-1}$

equating (2) and (4) and transposing terms

$$
W_{2}=W_{1}\left(\frac{\lambda_{2}}{\lambda_{1}}\right)^{4} \frac{\left(e^{\frac{\lambda_{2}}{\lambda_{2}}}-1\right)}{\left(e^{\frac{b}{\lambda_{1}}}-1\right)}
$$

To evaluate the constant $b, T$ is assumed to be $20,000^{\circ}$.

Tables $8-10$ show the results obtained first when it is assumed that $W_{1}$ equals $40 \%$ of the $2 P-4 D$ line, secondly when $w_{1}$ equals $30 \%$ of this line. All the other corrections that have been suggested are shown and applied. Figures $10-15$ are plots of the net equivalent widths against serial number.

Comparing the corrected values with the measured equivalent widths in giant stars, we see that a correction of $40 \%$ makes the first member of each series in the dwarf and intermediate stars much more intense than the corresponding line in a giant star of about the same spectral class It also makes the second member of the series less in the case of the dwarf and intermediate stars than it is in giant stars. The remaining lines are in general stronger in the dwarf and intermediate stars than in the giants. We would expect that in dwarf and internediate stars the first member of both the singlets and triplets should have much the same intensity as in giant stars, whereas the higher members should be somewhat 
Table 8.

$\gamma$ Pegasi

\begin{tabular}{|c|c|c|c|c|}
\hline & W & courection & & net $W$ \\
\hline \multirow[t]{2}{*}{6678} & .55 & plus $40 \%$ Fos ter leakage & $\$ .76$ & 1.31 \\
\hline & & plus $30 \%$ Foster leakage & +.58 & 1.13 \\
\hline \multirow[t]{2}{*}{4922} & .96 & Iess $40 \%$ Foster leakage & -.38 & .58 \\
\hline & & Iess $30 \%$ Foster leakage & -.29 & .67 \\
\hline 4388 & .82 & none & & $.8 \overline{2} 2$ \\
\hline 4144 & .79 & Iess sulphur blend & -.08 & .71 \\
\hline 4009 & .55 & none & & .55 \\
\hline \multirow[t]{2}{*}{5876} & .77 & plus $40 \%$ Foster leakage & +.90 & 1.67 \\
\hline & & plus $30 \%$ Foster leakage & +.67 & 1.44 \\
\hline \multirow[t]{2}{*}{4471} & 1.28 & less $40 \%$ Poster leakage & -.51 & .77 \\
\hline & & Iess $30 \%$ Foster leakage & -.38 & .90 \\
\hline 4026 & 1.25 & Iess 4024 blend & -.12 & 1.13 \\
\hline 3820 & 1.14 & none & & 1.14 \\
\hline
\end{tabular}

Table 9. Herculis

\begin{tabular}{|r|r|l|r|r|}
\hline & W & correction & net W \\
\hline 6678 & .47 & plus 40\% Foster leakage & +.72 & 1.19 \\
& & plus 30\% Foster leakage & +.54 & 1.01 \\
4922 & .91 & Iess $40 \%$ Foster leakage & -.36 & .55 \\
& & less 30\% Foster leakage & -.27 & .64 \\
4388 & .64 & none & & .64 \\
4144 & .62 & less sulphur blend & -.04 & .58 \\
4009 & .44 & & .44 \\
\hline
\end{tabular}


Table 9. (cont)

\begin{tabular}{|c|c|cc|c|}
\hline & W & correction & net W \\
\hline 5876 & .61 & plus $40 \%$ Foster leakage +.76 & 1.37 \\
& & plus 30\% Foste leakage +.56 & 1.17 \\
4471 & 1.07 & less $40 \%$ Foster leakage & -.43 & .64 \\
& & less 30\% Foster leakage & -.32 & .75 \\
4026 & 1.02 & less 4024 blend & -.10 & .92 \\
3820 & .93 & none & & .953 \\
\hline
\end{tabular}

Table 10. $\eta$ Ursae Majoris

\begin{tabular}{|c|c|c|c|c|}
\hline & $\mathrm{W}$ & correction & & net $W$ \\
\hline \multirow[t]{2}{*}{6678} & .54 & plus $40 \%$ Foster leakage & +.76 & 1.37 \\
\hline & & plus $30 \%$ Foster leakage & +.50 & 1.04 \\
\hline \multirow[t]{2}{*}{4922} & .85 & Iess $40 \%$ Foster leakage & -.34 & .51 \\
\hline & & Iess $30 \%$ Foster leakage & -.25 & .60 \\
\hline 4388 & .70 & none & & .70 \\
\hline 4144 & .66 & less sulphur blend & -.09 & .57 \\
\hline 4009 & .43 & noné & & .43 \\
\hline \multirow[t]{2}{*}{5876} & .80 & plus $40 \%$ Foster leakage & +.70 & 1.50 \\
\hline & & plus $30 \%$ Foster leakage & $9.53=$ & 1.33 \\
\hline \multirow[t]{2}{*}{4471} & 1.01 & Iess $40 \%$ Foster leakage & -.40 & .61 \\
\hline & & less $30 \%$. Foster leakage & -.30 & .71 \\
\hline 4026 & 1.28 & less 2024 blend & -.10 & 1.18 \\
\hline 3820 & .60 & none & & .60 \\
\hline
\end{tabular}




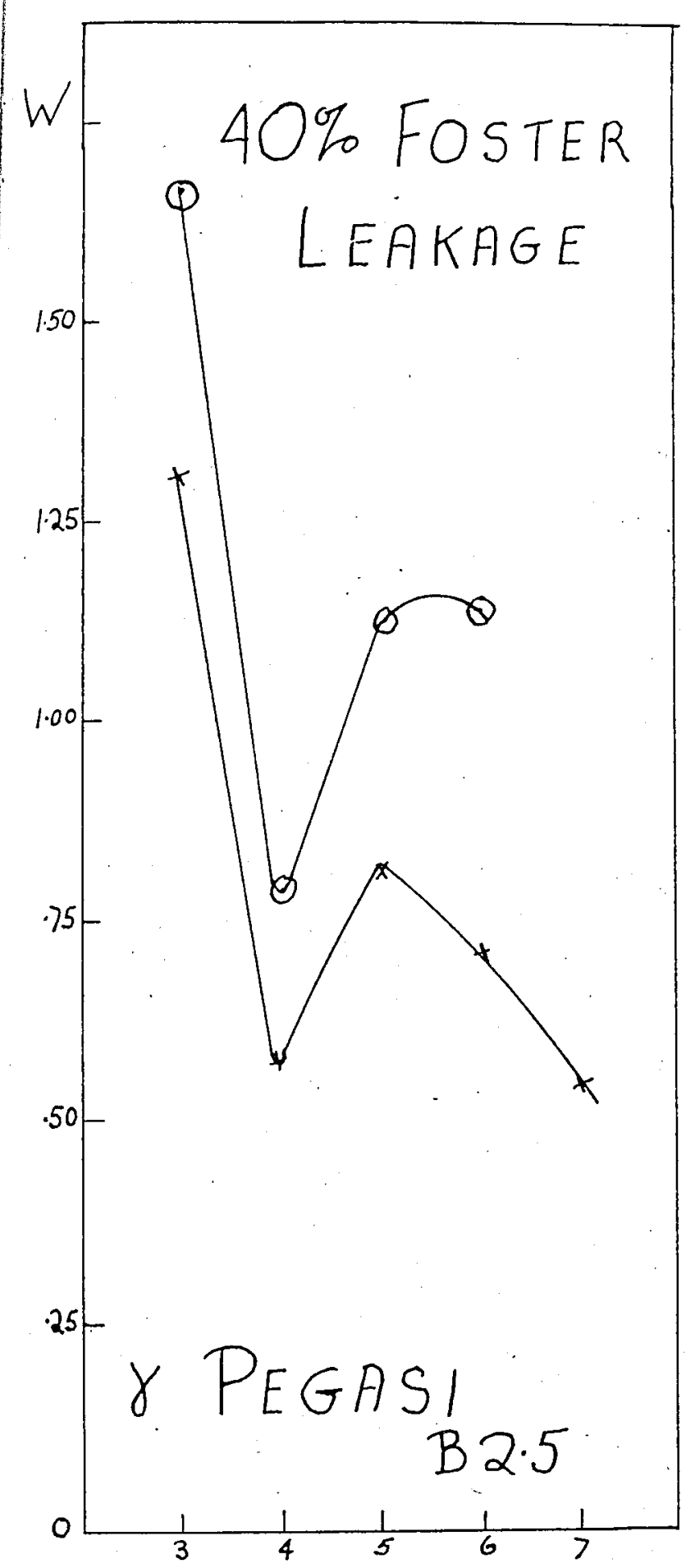

SERIAL No. $h$

fig. 10

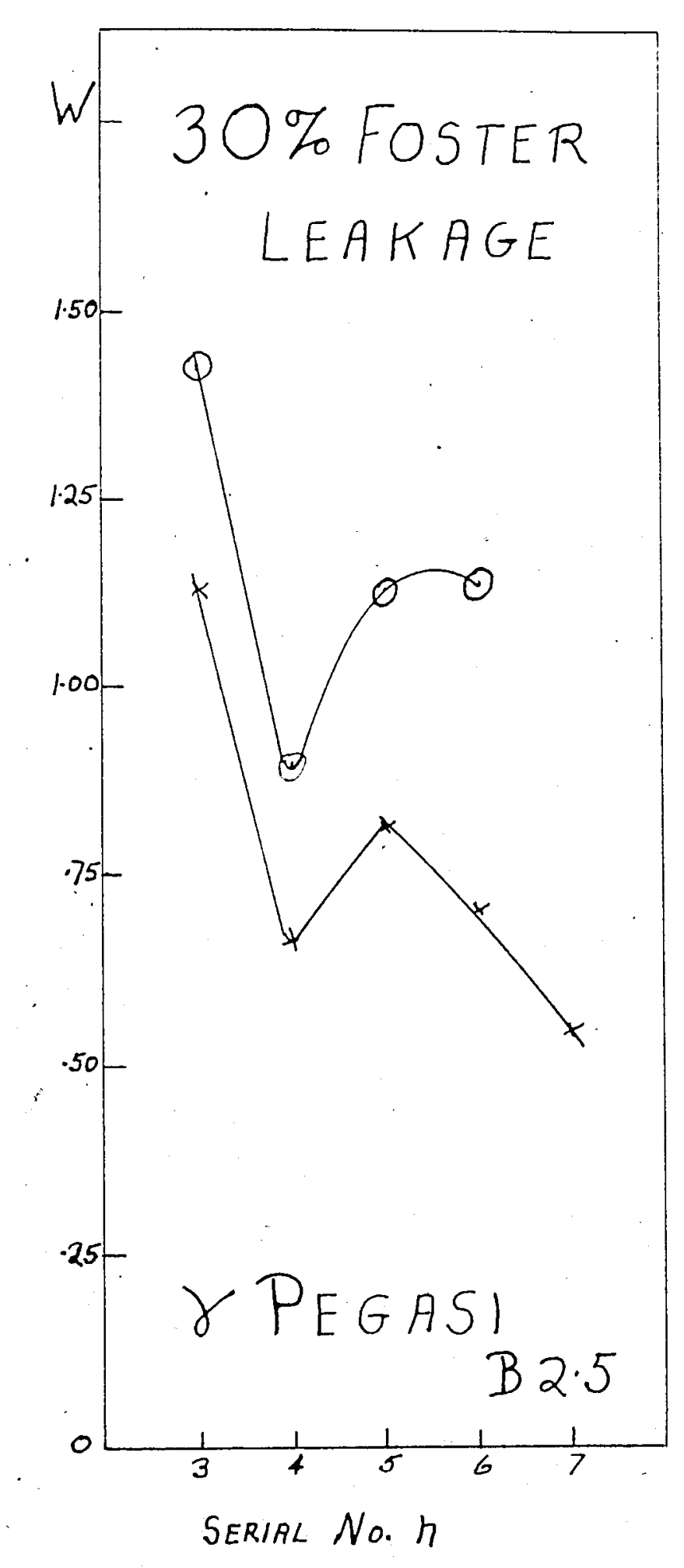

fig. 11 .

$$
\begin{aligned}
& X=S I N G L E T S \\
& O=\text { TRIPLETS }
\end{aligned}
$$




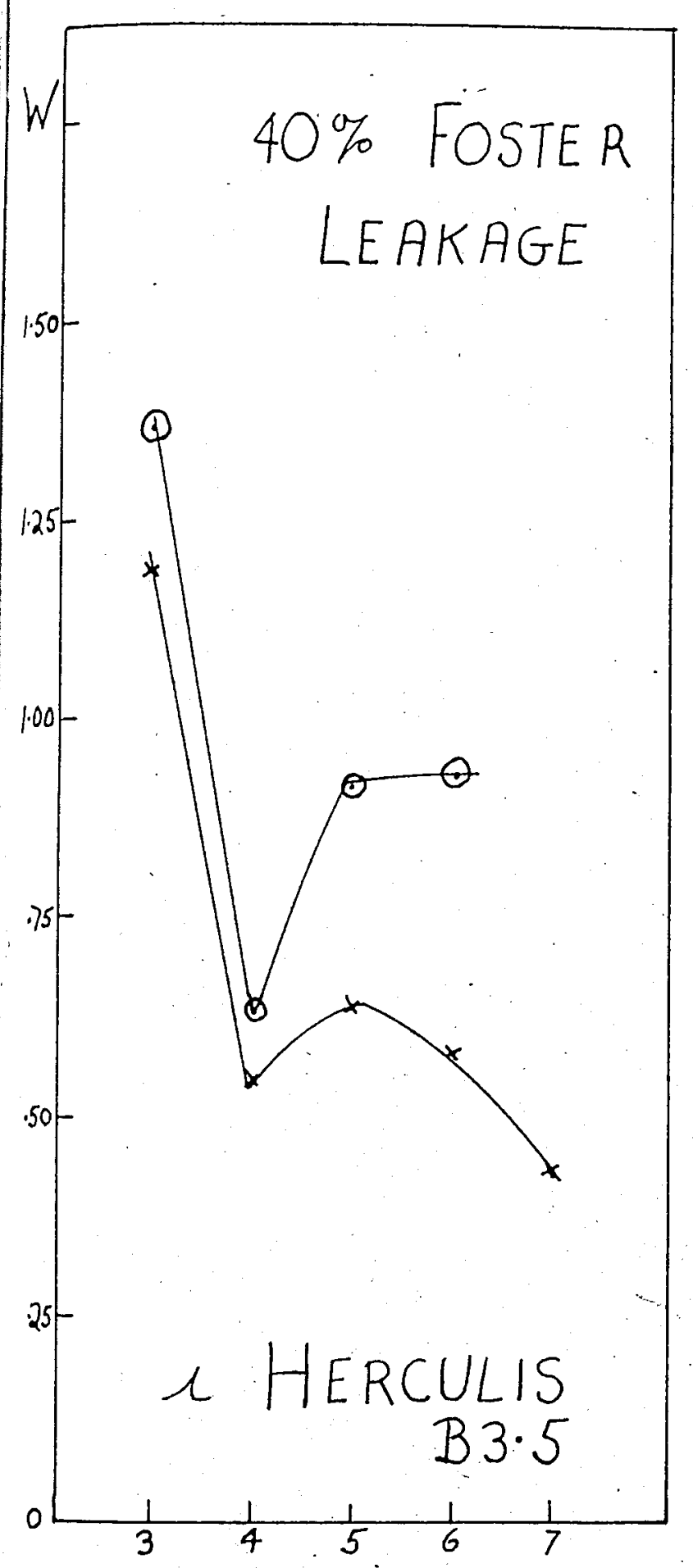

SERIRL No $h$

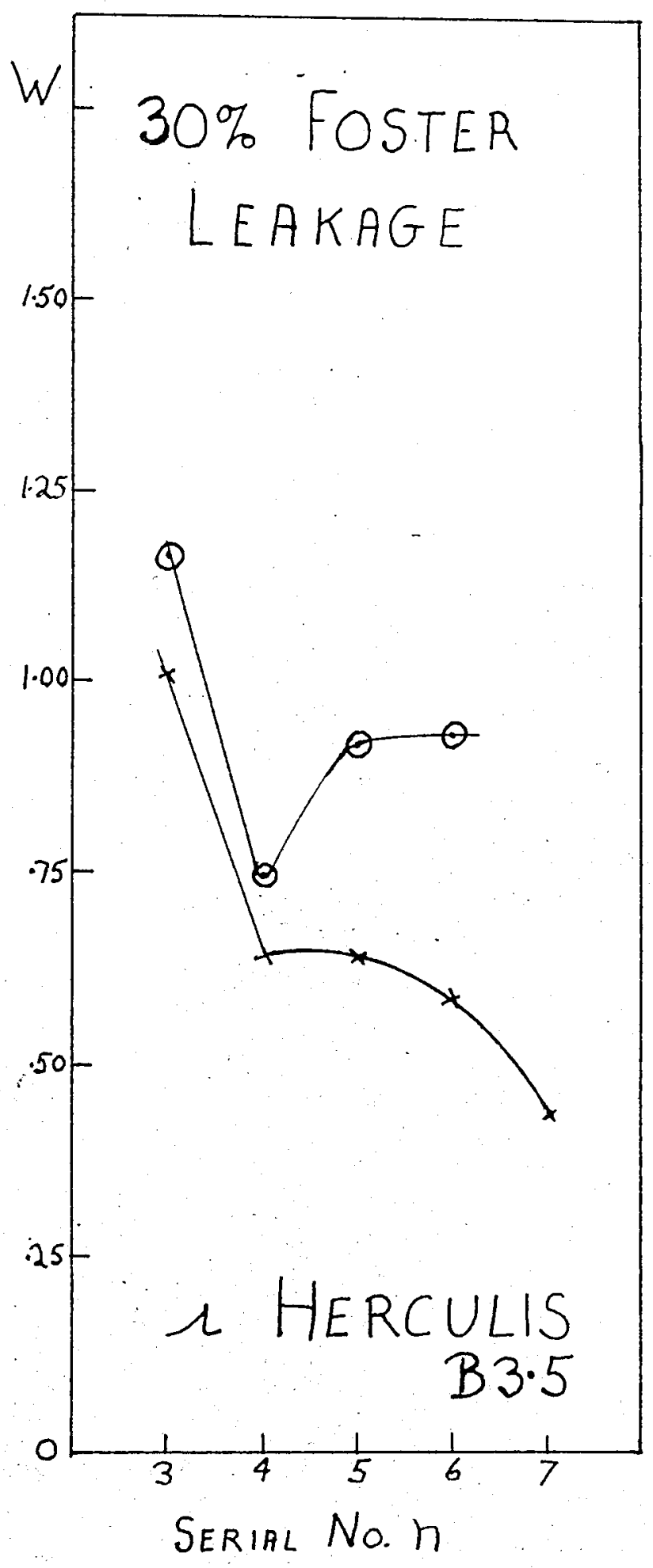

fig. 13.

fig. 12

$X=$ SINGLETS

$O=$ TRIPLETS 


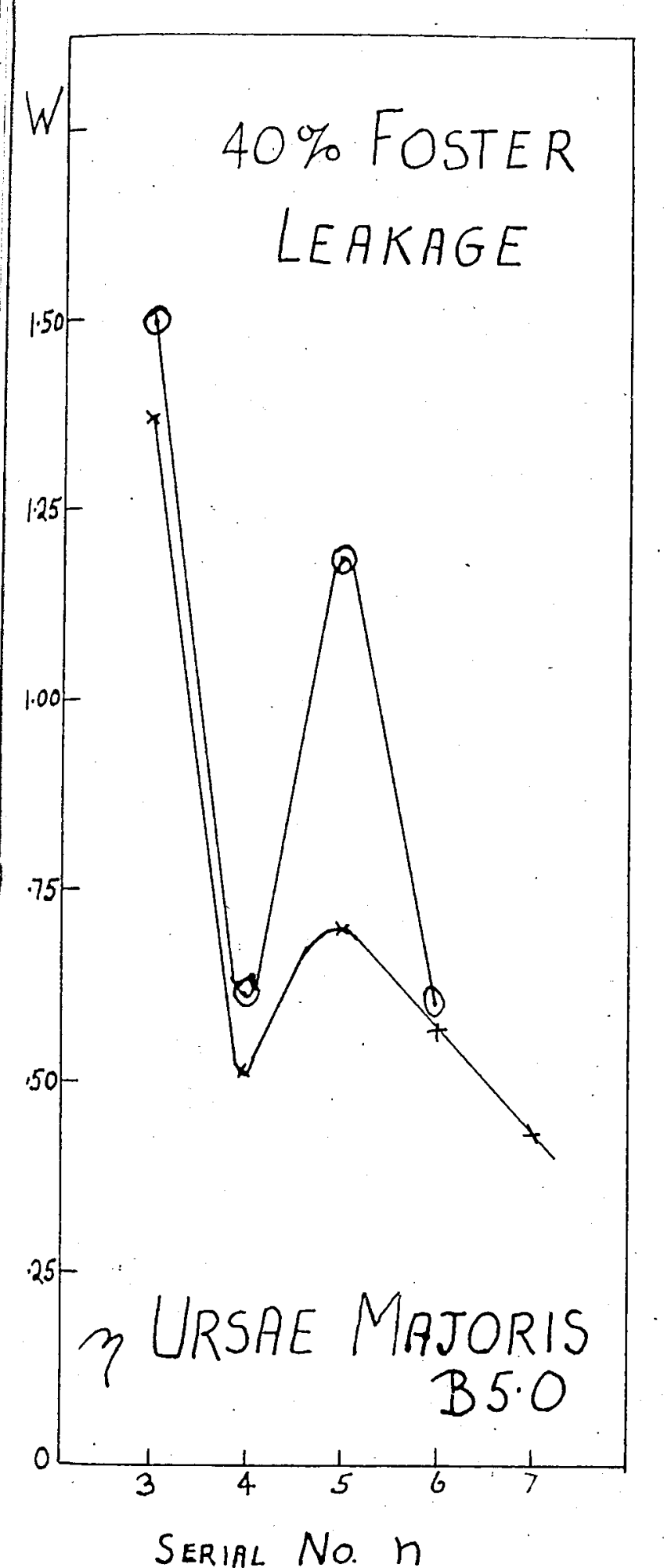

Serial No $\mathrm{h}$

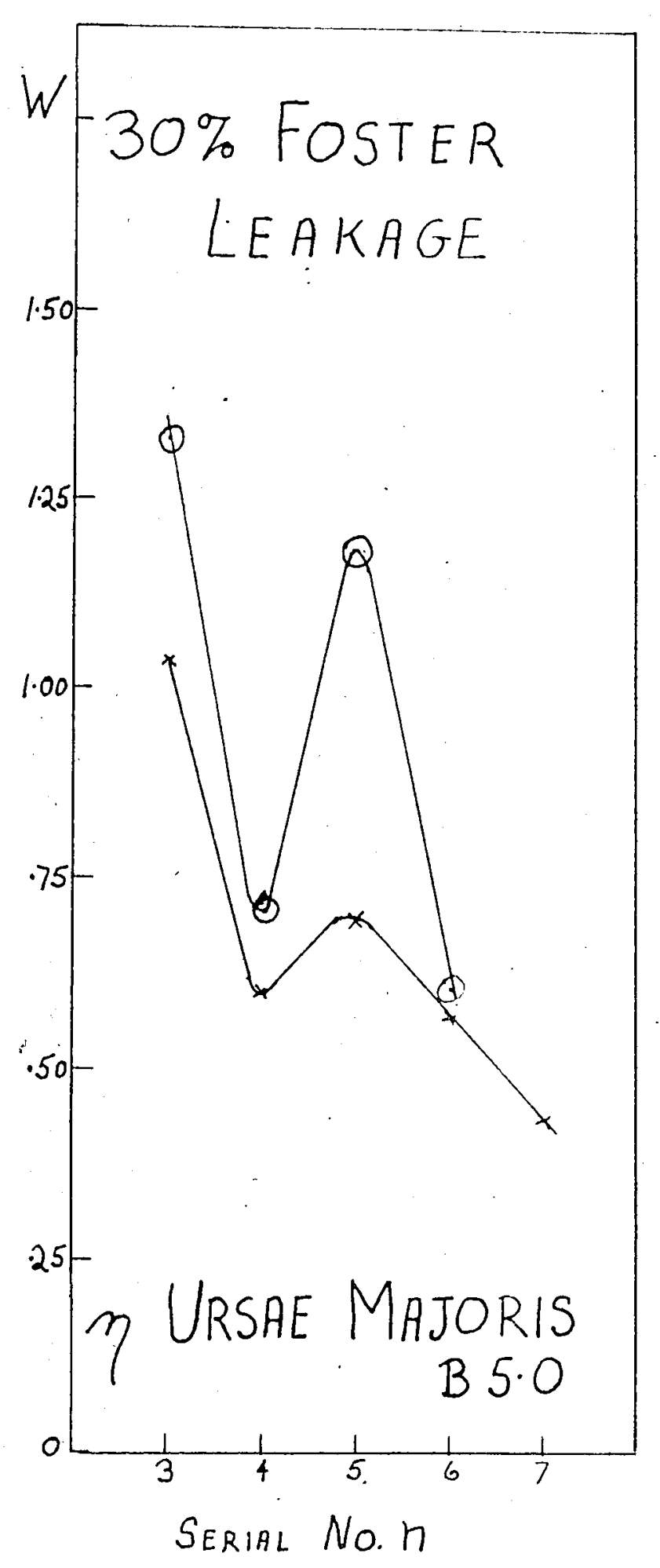

fig. 15

fig. 14 .

$X=$ SINGLETS

$\odot=$ TRIPLETS 
more intense in dwarfs and intermediates than in giants, since the effective Stark broadening increases with serial number.

It thus seems that a $40 \%$ correction raises the first member and lowers the second member of each series too far above and below a pure stark curve. If the correction is $30 \%$ of the 2P-4D line, then the results are much closer to the expected curve. In each case for the dwarf and intermediate stars, $\lambda 6678$ and $\lambda 5876$ have about the same intensity as in the giant wotars, while $\lambda 4922$ and $\lambda 4471$ are about the same, or even lower ta. than in giants.

Actually the leakage need not be very great to have an appreciable effect on the intensities of $\lambda 6678$ and $\lambda 5876$. Recalling that

$$
W_{2}=W_{1}\left(\frac{\lambda_{2}}{\lambda_{1}}\right)^{4} \frac{\left(e^{\frac{b}{\lambda_{2}}}-1\right)}{\left(e^{\frac{b}{\lambda_{t}}}-1\right)}
$$

and substituting in values for $b$ with $T=20,000^{\circ}$, and for $\lambda_{2}=6678, \lambda_{1}=4922$, it is seen that $w_{2}=2.00 w_{1}$ for the singlets, and for the triplets $W_{2}=1.76 W_{1}$. In other words a leakage of energy corresponding to an equivalent width of . 1 angstroms at $\lambda_{4922}$ results in an emission of energy of .2 equivalent angstroms at $\lambda 6678$, thus effectively weakening this line. A leakage of .1 equivalent angstroms at $\lambda 4471$ results in an emission of .176 equivalent angstroms at $\lambda 5876$.

Foster and Douglas estimated the leakage absorption to be over $40 \%$ of the 2P-4D line. However it seems more reasonable upon the above evidence to estimate this leakage as forming only about $30 \%$ of the line. The method used by Foster and Douglas in estimating the relative absorption of Stark components in the 
lines under consideration is by no means exact. Hence their results may be subject to considerable uncertainty.

Work done subsequent to writing this thesis has shown that the results given in the paper by Foster and Douglas (M.N., 22, 150, 1939) have been misinterpreted. When the area marked $X$, in the profiles of $\lambda 4471$ for YPegasi and cHerculis shown in this paper, and the total area, $Z$, of the line are compared it is found that $X$ equals about $30 \%$ of $Z$, in complete agreement with the results announced above. A careful reading of the paper by Foster and Douglas reveals that they claim $\frac{X}{2-x}=45 \%$ not $\frac{X}{Z}=45 \%$ as was as sumed in the above development. The solution of the equation $\frac{x}{2-x}=45 \%$ for $\frac{x}{Z}$ yielas the result that $\frac{X}{2}$ is about $30 \%$, a value found to be consistent with the reconstruction of the expected Stark Intensity curve. 


\section{Summary of Results:}

Data have been presented which support the hypothesis of a leakage cycle as proposed by Foster and Douglas to explain the strong absorption near the $2 \mathrm{p}-4 \mathrm{~d}$ Iines of HeI in the spectra of dwarf B type stars. However it seems likely that this absorption constitutes about $30 \%$ of the line rather than over $40 \%$ as proposed by Foster and Douglas. The hypotheses suggested by Struve and Goldberg to explain the absorption near $\lambda 4471$ aare not satisfactory, since both hypotheses indicate relatively no change in the strengths of the leading members of the diffuse series in giant and dwarf stars of the same spectral type, whereas a weakening of these lines has been observed.

An excessive absorption near $\lambda 4026$ has been found in dwarf stars. From the data examined no explanation of this effect can be offered. A further analysis of the spectra of class $B$ dwarf stars is requited before any statement on this point can be given.

I would like to acknowledge the kind assistance of alI members of the staff of the Dominion Astrophysical Observatory, Victoria, B.C., who most generously took plates of the stars studied, and contributed much to the progress of this investigation by many stimulating discussions of the problem. In particular I would like to thank Dr. R. M. Petrie, upon whose suggestion this investigation was undertaken, for his nany helpful suggestions concerning the problem, and for his guidance imselecting data. The analysis of the data and 
writing of the paper was concluded at the University of British Columbia under the direction of Dr. Wm. Petrie. 
The separate determinations of equivalent width for each line, together with the instrument used, are shown in the following tables 11-16. The wighted mean equivalent width for each line with the proballe error is given at the bottom of each column. The assigned weights are indicated first in each column, then the equivalent width of the line in angstroms. The weights are relative, and depend on the dispersion and actual plate features.

Table 11

Leonis

\begin{tabular}{|c|c|c|c|c|c|}
\hline \multicolumn{6}{|c|}{ Diffuse Singlets } \\
\hline & $\begin{array}{l}2^{1} \mathrm{P}-3^{1} \mathrm{D} \\
6678.149\end{array}$ & $\begin{array}{l}2^{1} \mathrm{P}-4^{1} \mathrm{D} \\
4921.929\end{array}$ & $\begin{array}{l}2^{1} P-5^{1} D \\
4387.928\end{array}$ & $\begin{array}{l}2^{1} \mathrm{P}-6^{1} \mathrm{D} \\
4143.759\end{array}$ & $\begin{array}{l}2^{1} \mathrm{P}-7^{I_{D}} \\
4009.270\end{array}$ \\
\hline III M & 1.02 & 2 & & & \\
\hline$I M$ & & .46 & .43 & .37 & .17 \\
\hline & & 1.44 & 1.30 & .35 & .20 \\
\hline & & 1.52 & 1.35 & .19 & .25 \\
\hline & 1.02 & $.47 \pm .01$ & $.36 \pm .03$ & $.30 \pm .04$ & $.21 \pm .01$ \\
\hline
\end{tabular}




\begin{tabular}{|c|c|c|c|c|}
\hline \multicolumn{2}{|c|}{ Table 11 (cont). } & \multicolumn{2}{|c|}{ Diffuse Triplet } & \\
\hline & $2^{3} \mathrm{P}-3^{3} \mathrm{D}$ & $2^{3} \mathrm{P}-4^{3} \mathrm{D}$ & $2^{3} \mathrm{P}-5^{3} \mathrm{D}$ & $2^{3} P-6^{3} D$ \\
\hline & 5875.618 & 4471.477 & 4026.189 & 3819.606 \\
\hline GII L & $3 \quad 1.29$ & & & \\
\hline III M & 11.10 & & & \\
\hline & 11.00 & & & \\
\hline I M & & .86 & .46 & .23 \\
\hline & & 10.63 & 1.59 & .45 \\
\hline & & $1 \quad .63$ & 11.48 & $1 \quad .32$ \\
\hline & $1.19 \pm .03$ & $.71 \pm .05$ & $.51 \pm .03$ & $.33 \pm .05$ \\
\hline
\end{tabular}

Table 12

55 Cygni

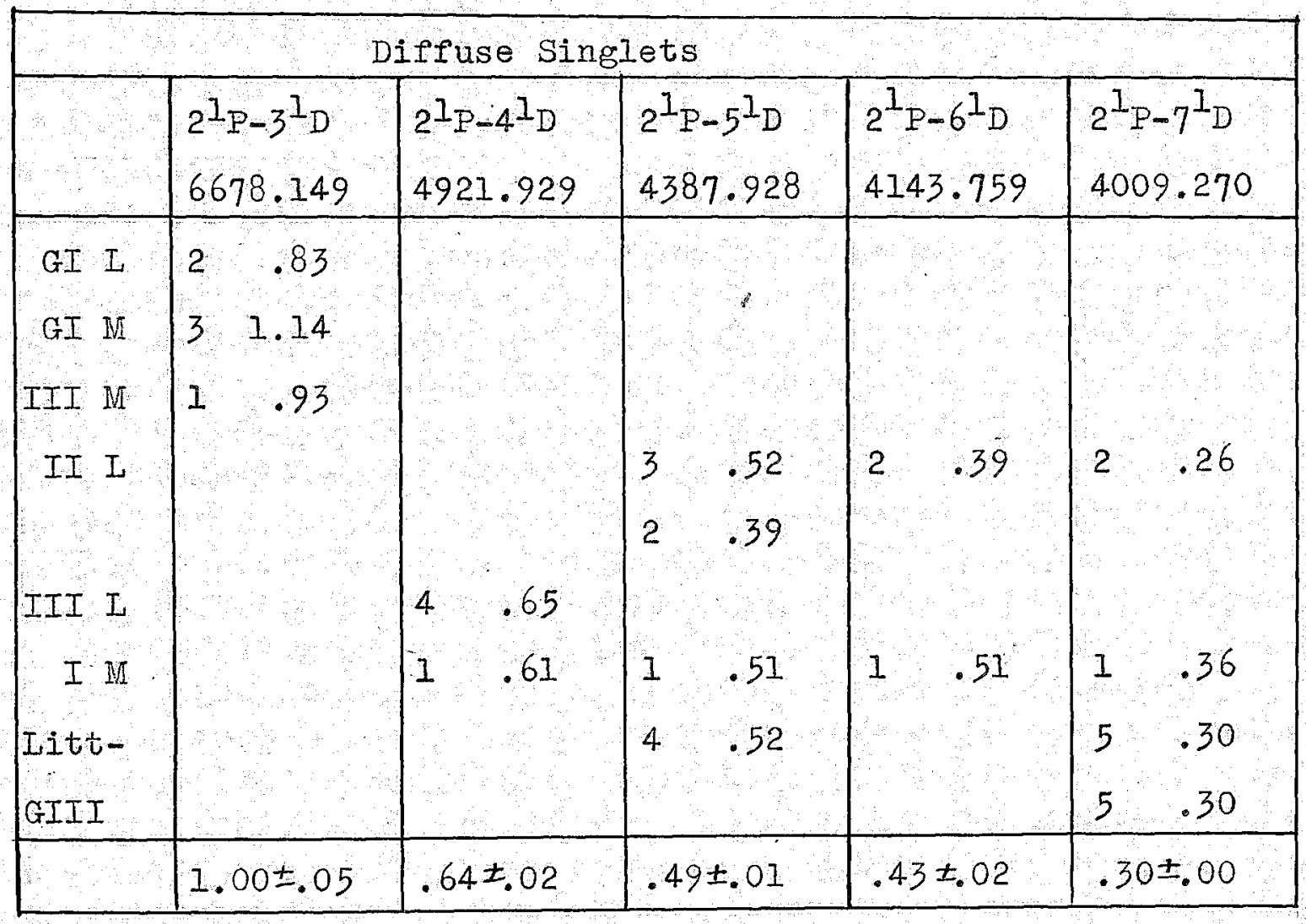


31.

\begin{tabular}{|c|c|c|c|c|}
\hline \multicolumn{2}{|c|}{ Table 12 (oont.) } & \multicolumn{2}{|c|}{ Diffuse Triplets } & \\
\hline & $\begin{array}{l}2^{3} \mathrm{P}-3^{3} \mathrm{D} \\
5875.618\end{array}$ & $\begin{array}{l}2^{3} \mathrm{P}-43 \mathrm{D} \\
4471.477 \\
\end{array}$ & $\begin{array}{l}2^{3} P-5^{3} D \\
4026.189 \\
\end{array}$ & $\begin{array}{l}2^{3} \mathrm{P}-6^{3} \mathrm{D} \\
3819.606 \\
\end{array}$ \\
\hline GI L & 3.76 & & & \\
\hline & $3 \quad .89$ & & & \\
\hline III M & $1 \quad .89$ & & & \\
\hline IIII & & $\begin{array}{ll}3 & .81 \\
2 & .76\end{array}$ & $2 \quad .51$ & \\
\hline I M & & $1 \quad .52$ & $\begin{array}{ll}1 & .62 \\
1 & .66\end{array}$ & 1.56 \\
\hline Litt- & & & .64 & .56 \\
\hline GIII & & & $3 \quad .67$ & $4 \quad .57$ \\
\hline : & $.83 \pm .02$ & $.74 \pm .03$ & $.62 \pm .02$ & $.56 \pm .00$ \\
\hline
\end{tabular}

Table 13

67 ophiuchi

\begin{tabular}{|c|c|c|c|c|c|}
\hline \multicolumn{6}{|c|}{ Diffuse Singlets } \\
\hline & $\begin{array}{l}2^{I} \mathrm{P}-3^{1} \mathrm{D} \\
6678.149\end{array}$ & $\begin{array}{l}2^{I_{P}-4^{I_{D}}} \\
4921.929\end{array}$ & $\begin{array}{l}2^{I_{P}-5^{I} D} \\
4387.928\end{array}$ & $\begin{array}{l}2^{1} P-6^{1} D \\
4143.759\end{array}$ & $\begin{array}{l}2^{I_{\mathrm{P}}-7^{1} \mathrm{D}} \\
4009.270\end{array}$ \\
\hline III $R$ & $1 \quad 1.19$ & $4 \quad .81$ & & & \\
\hline & $1 \quad 1.12$ & $4 \quad .66$ & & & \\
\hline II L & & 4 & .44 & 4. .51 & .36 \\
\hline & & & .39 & 4.44 & 4.39 \\
\hline & & & & $4 \quad .43$ & $4 \quad .28$ \\
\hline I M & & .81 & .42 & .44 & .37 \\
\hline & & .70 & .49 & 1.44 & .31 \\
\hline Litt-II & & .79 & $1 \quad .32$ & .35 & $1 \quad .29$ \\
\hline & $1.16 \pm .03$ & $.74 \pm .02$ & $.41 \pm .01$ & $.45 \pm .01$ & $.34 \pm .01$ \\
\hline
\end{tabular}


32.

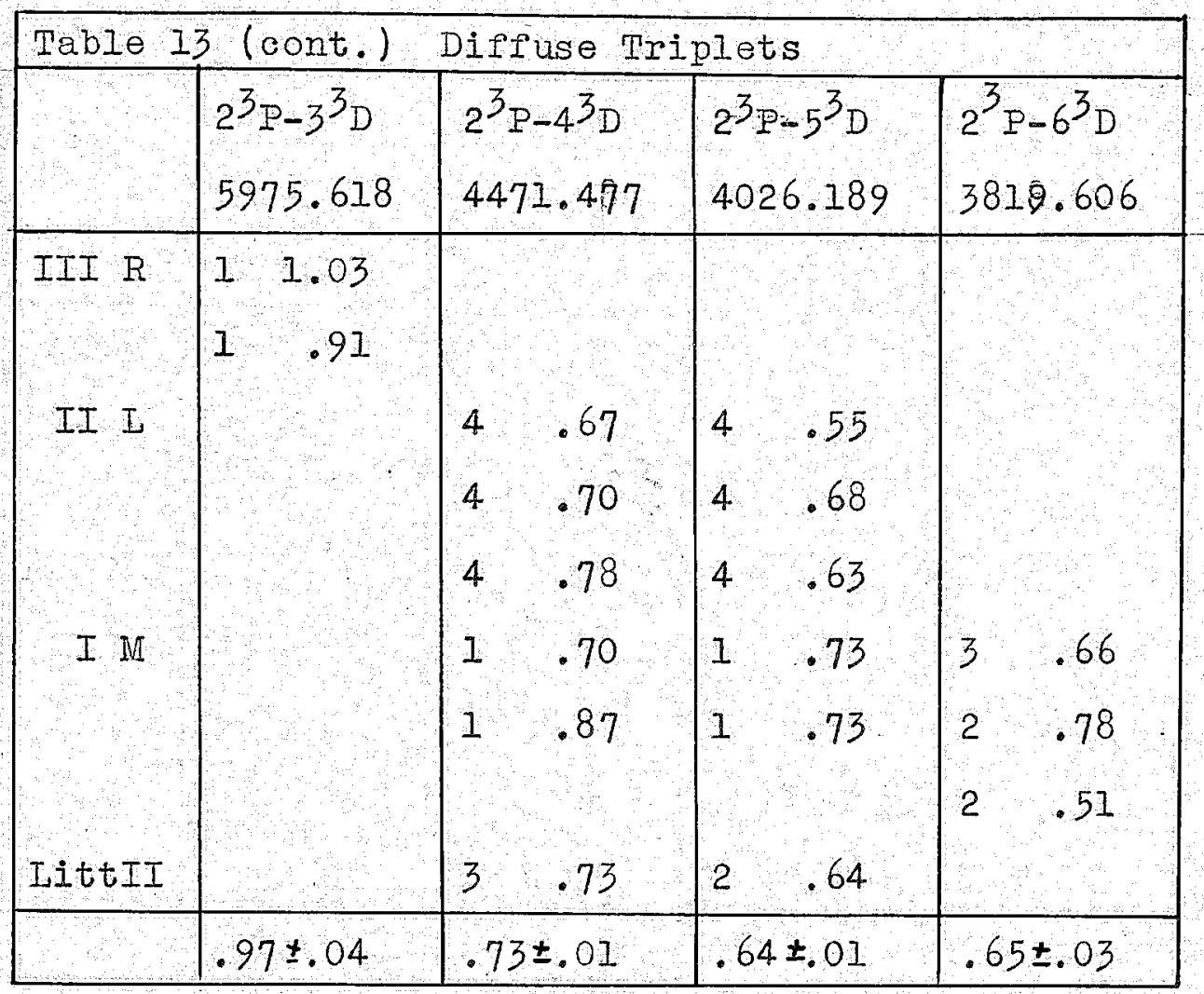


Table 14

$\gamma$ Pegasi

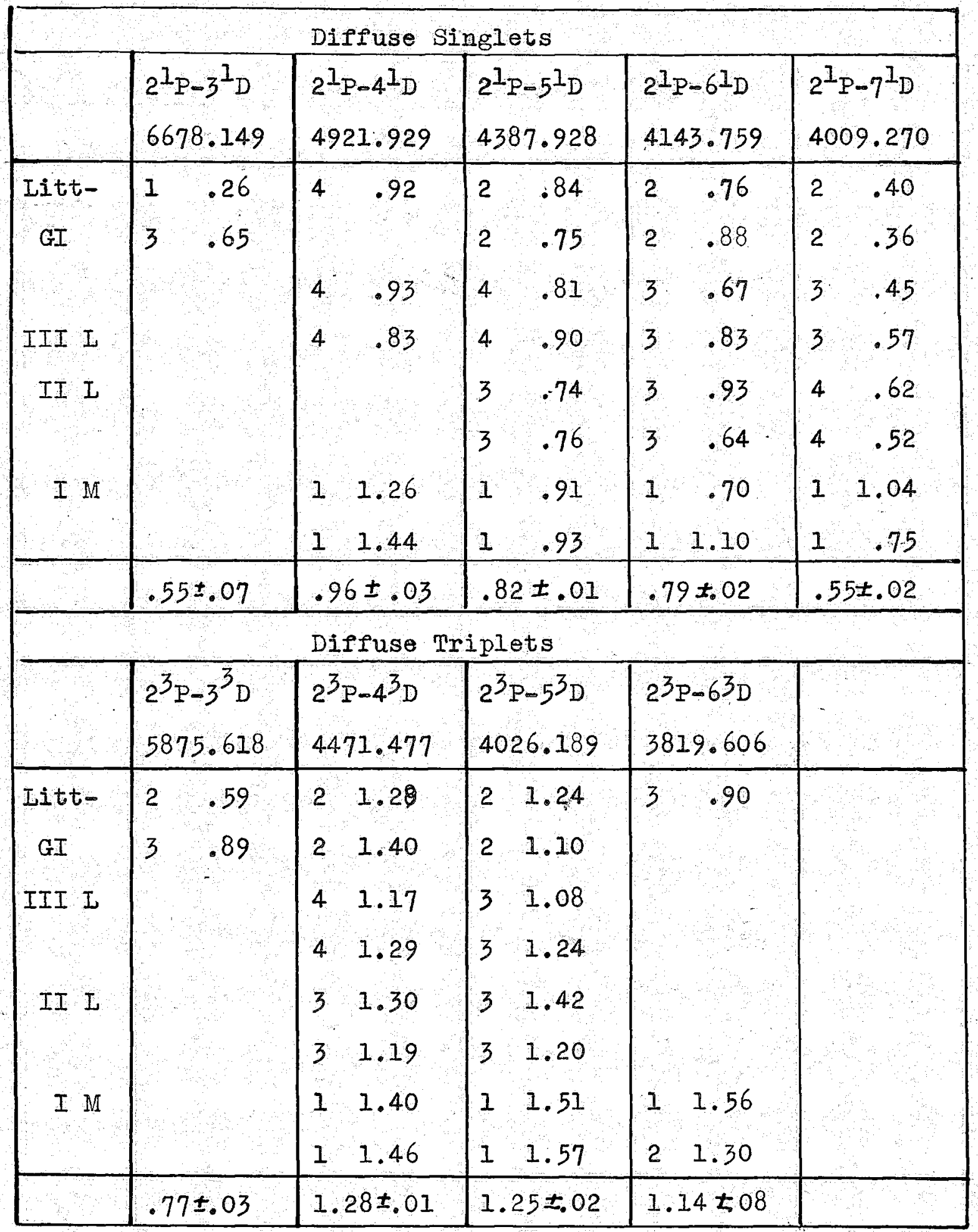


Table 15.

eHerculis

\begin{tabular}{|c|c|c|c|c|c|c|c|c|}
\hline \multicolumn{9}{|c|}{ Diffuse Singlets } \\
\hline & $\begin{array}{l}2^{I_{\mathrm{P}}-3^{-}} \mathrm{D} \\
6678.149\end{array}$ & $\begin{array}{l}2^{I} \mathrm{P}-4^{1} \mathrm{D} \\
4921.929\end{array}$ & & $\begin{array}{l}5^{I \mathrm{D}} \\
.928\end{array}$ & & $\begin{array}{l}.6^{1} \mathrm{D} \\
.759 \\
\end{array}$ & & $\begin{array}{l}7^{1} \mathrm{D} \\
.270\end{array}$ \\
\hline I M & & $\begin{array}{lr}1 & .70 \\
1 & 1.15\end{array}$ & $\begin{array}{l}3 \\
3\end{array}$ & $\begin{array}{l}.74 \\
.67 \\
.62 \\
.65 \\
.64 \\
.69 \\
.55 \\
.58 \\
.68 \\
.64 \\
.69 \\
.58 \\
.57 \\
.76 \\
.62 \\
.60 \\
.60 \\
.58 \\
.66 \\
.64 \\
.88 \\
.58 \\
.68 \\
.68 \\
.69\end{array}$ & $\begin{array}{l}I \\
I\end{array}$ & $\begin{array}{l}.70 \\
.65 \\
.47 \\
.60\end{array}$ & $\begin{array}{l}3 \\
3 \\
3 \\
3\end{array}$ & $\begin{array}{l}.44 \\
.51 \\
.45 \\
.35\end{array}$ \\
\hline
\end{tabular}


Table 15 (cont)

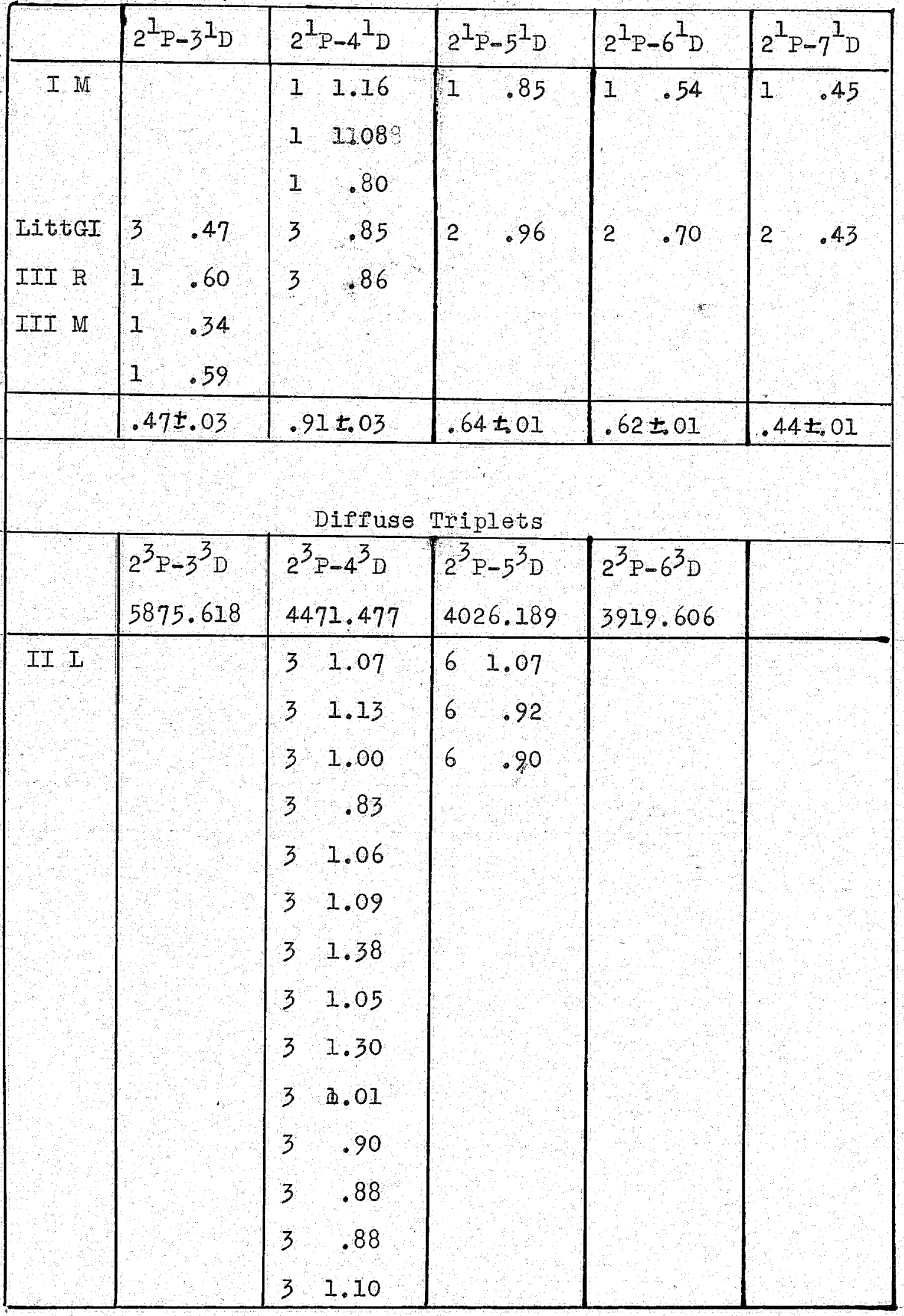


36.

Table 15 (cont)

\begin{tabular}{|c|c|c|c|c|}
\hline & $2^{3} \mathrm{P}-3^{3} \mathrm{D}$ & $2^{3} \mathrm{P}-4^{3} \mathrm{D}$ & $2^{3} P-5^{3} D$ & $2^{3} P-6^{3} D$ \\
\hline \multirow[t]{7}{*}{ II I } & & 31.06 & & \\
\hline & & $3 \quad 1.52$ & & \\
\hline & & 31.03 & & \\
\hline & & 31.16 & & \\
\hline & & $\begin{array}{ll}3 & .97\end{array}$ & & \\
\hline & & 3.91 & & \\
\hline & & 2.95 & & \\
\hline \multirow[t]{4}{*}{ I $\mathbb{M}$} & & 11.23 & 21.15 & .96 \\
\hline & & 11.19 & $\begin{array}{ll}2 & 1.17\end{array}$ & $1 \quad 1.12$ \\
\hline & & 11.30 & $\begin{array}{ll}1 & 1.13\end{array}$ & .93 \\
\hline & & & & 1.93 \\
\hline IittGI & .58 & 21.20 & 31.10 & .73 \\
\hline III $\mathrm{R}$ & .76 & & & \\
\hline \multirow[t]{2}{*}{ III $\mathrm{M}$} & .55 & & & \\
\hline & $1 \quad .61$ & & 8 & \\
\hline & $1.61 \pm .02$ & $1.07 \pm .01$ & $1.02 \pm .01$ & $.93 \pm .04$ \\
\hline
\end{tabular}


37.

Table 16

3 Ursae Majoris

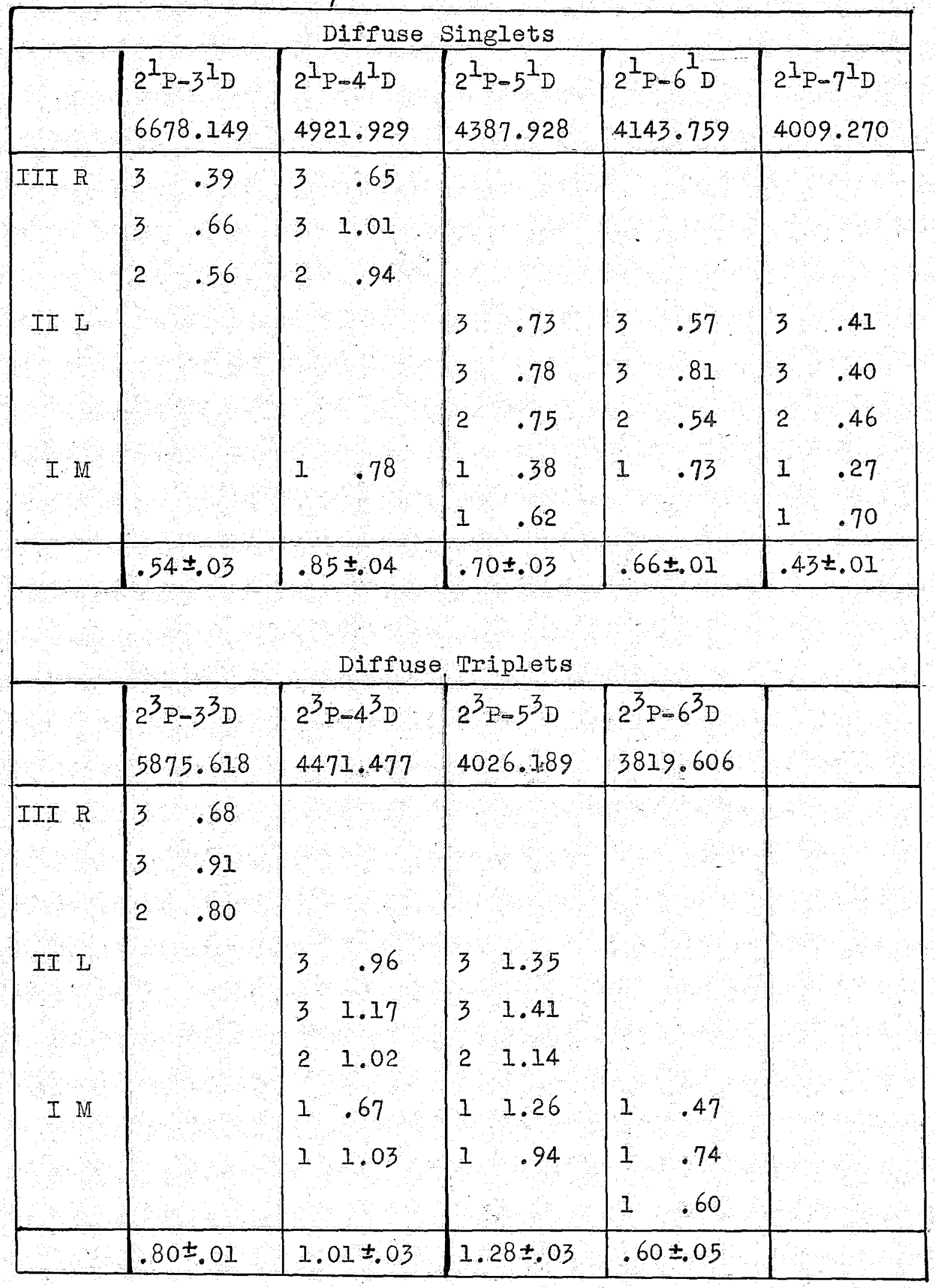




\section{BIBLIOGRAPHY}

Elvey, C.T., Struve, 0., Ap. J. 12, 277, 1930. Foster, J.S., Proc. Roy. Soc. 117, 137, 1927 Foster, J.S., Douglas, A.V., Nature 134, 417, 1934. M.N. $29,150,1939$.

Goldberg, Leo, Ap. J. 89, 623, 1939.

$$
\text { Ap. J. 20, 414, } 1939 .
$$

Merrill, F.W., Adams, W.S., Ap. J. 27, 98, 1943.

Moore, C.E., A Multiplet Table of Astrophysical Interest. Princeton, N.J., Princeton University observatory, 1933.

Plaskett, H.H., Pub. D.A.O. I, 325, 1922.

Rosseland̄, S., Ap. J. 63, 218, 1926.

Struve, 0., Ap. J. 69, 173, 1929.

Ap. J. 20, 85, 1929.

Ap. J. 70, 237, 1929.

Ap. J. 14, 225, 1931.

Observatory 61, 53, 1938.

Unsold, A, Zeit. f. Astrophys. 21, 22, 1941.

Williams, E.G., Ap. J. 83, 279, 1936.

Ap. J. 83, 305, 1936.

Woolley, R.R., Observatory $60,235,1937$. 